\title{
CONTEXTOS LÍTICOS DE ASENTAMIENTOS ARCAICOS EN LA COSTA DE TALTAL (II REgIÓN, CHILE)
}

\author{
Patricio Galarce C. ${ }^{1}$ y Gabriela Santander H.
}

\section{* Introducción}

\section{Resumen}

Se presenta un análisis e interpretación de dos contextos líticos arcaicos situados en la costa árida del norte de Chile, en la localidad

de Taltal (Región de Antofagasta). Uno de los sitios (228/230), corresponde a un asentamiento fechado alrededor de 5000 años AP, mientras que el otro (226-5), permitió obtener fechas que lo sitúan en momentos anteriores a 10.000 años $\mathrm{AP}$, constituyendo unas de las fechas más tempranas para esta parte de la costa del Norte Grande

de Chile Los conjuntos artefactuales se caracterizan por intensas actividades de manufactura y utilización de instrumentos líticos, en el contexto de estrategias logísticas y residenciales de movilidad y uso del territorio en el espacio costero y ámbitos territoriales cercanos (p.e., pampas interiores), procesos enfocados en el aprovechamiento de recursos en dicha localidad.

Palabras claves: cazadores recolectores - contextos líticos - materias primas de alta calidad - tecnología bifacial - movilidad - asentamiento costa árida - Taltal - norte de Chile.

We present an analysis and interpretation from two archaic lithic contexts located in the arid coast of Northern Chile (Taltal, II region). $228 / 230$ site was dated around 5000 years $\mathrm{BP}$ and $226-5$ site was dated prior to 10.000 years BP, giving the earliest dates for human occupations from coastal Northern Chile. Artifactual assemblages suggest the development of intense stone tool making and wear activities in the context of logistical and residential mobility and land -use patterns in coastal and surrounding inner areas (pampas), through processes focused in resource utilization from this locality.

Key words: hunter gatherers - lithic contexts - high quality raw materials bifacial technology - mobility - settlement - arid coast - Taltal - Chile.

Recibido: julio 2011. Aceptado: abril 2013.
La localidad costera de Taltal, ubicada en el sector sur de la Región de Antofagasta, es conocida desde principios del s. XX por la riqueza de los contextos arqueológicos allí registrados (Uhle 1916; Oyarzún 1917; Capdeville 1921). Estos primeros trabajos versaron acerca de la discusión que sostenían los expertos respecto a la posibilidad de encontrar herramientas paleolíticas en el área, de acuerdo con las semejanzas tipológicas del material lítico de Taltal con piezas registradas en contextos del Paleolítico Europeo. Aunque esta discusión ha sido ampliamente superada por investigaciones posteriores (Bird 1988 [1943]; Berdichewsky 1962; Núñez 1984), ha quedado claro que la ocupación de la zona por parte de grupos cazadores recolectores arcaicos o precerámicos fue muy intensa y notoria por la extraordinaria calidad de los materiales recuperados.

Pese a lo anterior, muy poco trabajo arqueológico se ha enfocado en la caracterización sistemática de las ocupaciones arcaicas en el área de Taltal, tanto desde el punto de vista cronológico como contextual. Resulta paradójico que, frente a contextos de indudable valor científico, se aprecie baja preocupación de los investigadores por ellos, asistiendo de manera pasiva a la destrucción y pérdida de importante cantidad de información por la acción desmedida y sin control de aficionados y de quienes lucran con la venta de objetos arqueológicos, lamentablemente descontextualizados.

\footnotetext{
${ }^{1}$ Archeos Chile Consultores en Arqueología, Av. Walker Martínez 673, La Florida, Santiago, CHILE, E-mail: patogalarce@yahoo.es, gsantanderh@gmail.com
} 
Afortunadamente, en la última década esta situación ha tendido a cambiar gracias a estudios de impacto ambiental (EIA) derivados de las obras de mejoramiento para la carretera costera entre las localidades de Taltal y Caleta Paposo, los cuales han permitido identificar, registrar e intervenir un par de sitios arcaicos con contextos relativamente intactos (Figura 1). Lo anterior permite intentar una primera aproximación sistemática a las ocupaciones humanas del área, tanto en términos cronológicos como de características contextuales del registro arqueológico de esta localidad del Norte Grande (Castelleti et al. 2004, 2010; Castelleti y Maltrain 2010).

En esta lógica, el trabajo realizado en los sitios arqueológicos $228 / 230$ y $226-5$, datados en diferentes momentos del período Arcaico, nos posibilita decir algo más respecto a las poblaciones cazadoras recolectoras que ocuparon la zona, más allá de discutir respecto a simples tipologías de instrumentos diagnósticos, enfatizando la conformación de los conjuntos líticos a partir de factores tecnológicos, funcionales y de organización espacial de las actividades, siendo la intención principal del este escrito.

Cabe destacar la importancia de estos sitios en lo que se refiere al posicionamiento cronológico de los mismos, considerando que en Taltal existe un déficit de dataciones. De este modo, el sitio 228/230 se sitúa en momentos transicionales entre los períodos Arcaico Medio y Arcaico Tardío. Por su parte, el sitio 226-5, se ubica en tiempos del Arcaico Temprano, de forma contemporánea a sitios costeros áridos como La Chimba 13, al norte de Antofagasta y El Obispo 1, al sur de Chañaral (Llagostera 1979; Llagostera et al. 2000) y semiáridos como El Teniente, Huentelauquén y Punta Ñagué (Jackson 1993; Llagostera et al. 2000; Weisner et al. 2000).

\section{* Los conjuntos líticos: Características y METODOLOGÍa DE APROXIMACIÓN}

En contextos arqueológicos de cazadores recolectores prehistóricos, tal vez uno de los ítemes materiales más frecuentes e importantes son los artefactos líticos. El estudio sistemático de estos restos permite generar diversas aproximaciones para interpretar las conductas culturales de los grupos humanos al interactuar con su medio social y ecológico. Un primer aspecto a considerar apunta a inferir algunos de los procesos que inciden en la distribución estratigráfica y espacial de los materiales líticos que conforman un determinado contexto, así como la organización de las actividades involucradas con la manufactura y utilización de los instrumentos.

Debemos destacar que los conjuntos trabajados presentan características notables en la conformación de las actividades de producción lítica representadas en ellos. En primer lugar, ambos contextos exhiben una alta presencia de trabajo lítico reflejada en la depositación de ingentes cantidades de restos. Esta intensidad, muestra la representación de diversas actividades de elaboración y utilización instrumental, con un rol fundamental del empleo de materias primas silíceas y silicificadas de alta calidad, las cuales se transformaron en el soporte tecnológico de los grupos que ocuparon dichos lugares en el pasado. Por último, la utilización extensiva de materias primas de alta calidad permitió configurar contextos caracterizados por la elaboración de instrumentos de acabada terminación, donde incluso resulta posible observar aspectos tecnológicos relacionados con el aprendizaje de la talla lítica (Galarce 2008).

Con estos problemas en mente, se diseñó una estrategia de aproximación y análisis de los instrumentos y derivados de talla, en la cual se enfatiza la evaluación de variables distribucionales, tecnológicas y funcionales de estos conjuntos. Para el caso de los instrumentos, principalmente tallados, se realizó un análisis de clasificación con atributos morfofuncionales y tecnológicos (Bate 1971; Piel-Desruisseaux 1989; Jackson 1991; Shott 1993; Andrefsky 1998). Respecto a los derivados líticos, nuestra estrategia analítica apunta a discriminar variables distribucionales y tecnológicas de los conjuntos, que nos permitan establecer el comportamiento y orientación tecnológica de la producción de herramientas dentro de cada asentamiento (Sullivan y Rozen 1985; Andrefsky 1998; Bradbury y Carr 1999; Cornejo y Galarce 2010; De Souza 2003; Galarce 2004 y 2005).

Nuestra expectativa es la generación de mayor conocimiento acerca de cómo las comunidades cazadoras recolectoras que ocuparon la costa desértica, solucionaron problemas relacionados con la implementación de una tecnología instrumental y su utilización en el espacio y el tiempo. Al respecto, solucionada de forma relativa la ubicación cronológica de los sitios, podemos evaluar 


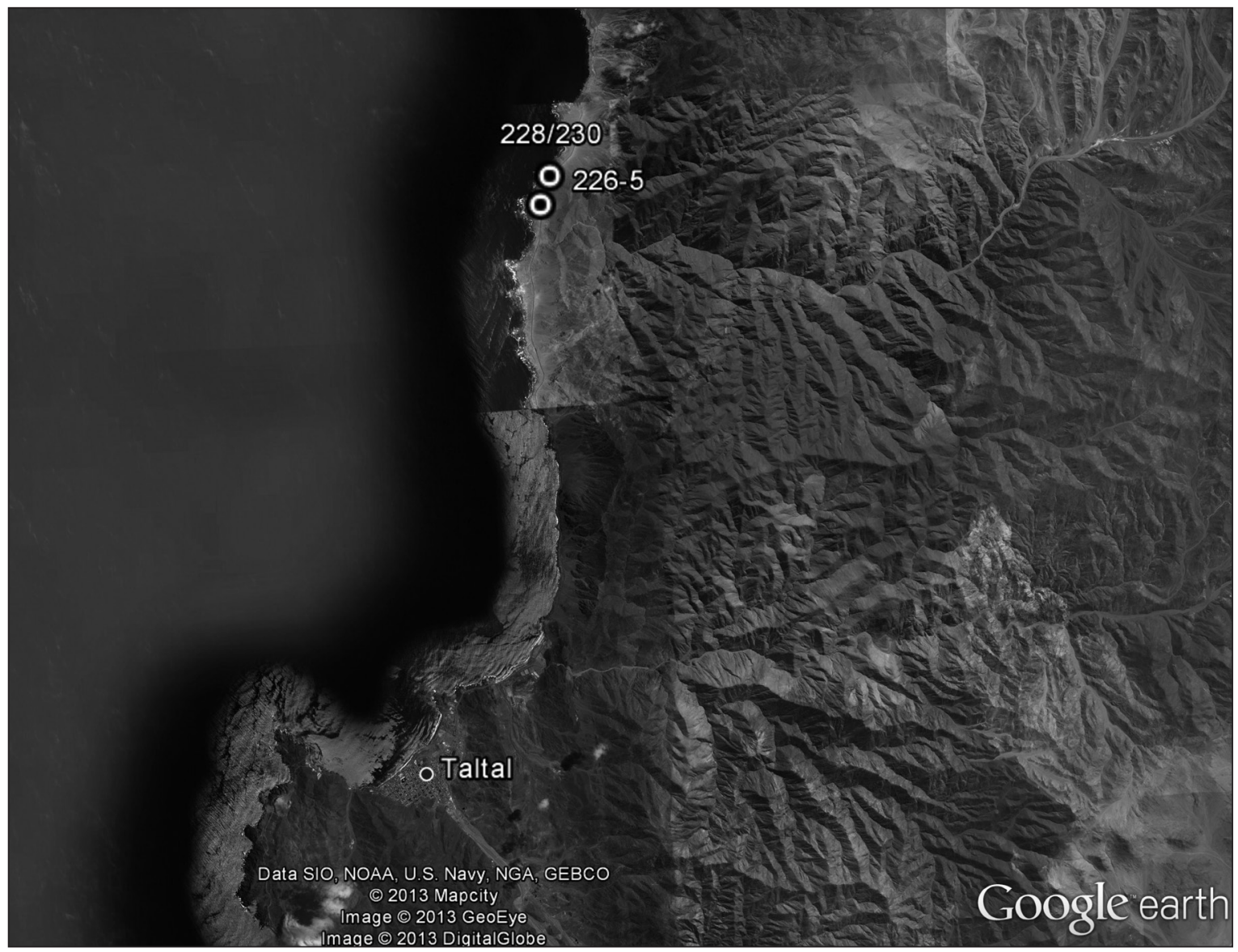

Figura 1. Ubicación de los sitios 226-5 y 228/230, en la costa al norte de Taltal (II Región).

también qué cambios o variaciones temporales pueden manifestarse en las formas que los grupos organizan la tecnología lítica durante su permanencia en los territorios que conforman nuestra área de estudio.

\section{* Caracterización y análisis DE LOS CONJUNTOS}

\section{Sitio 228/230}

Corresponde a un campamento abierto asociado a afloramientos rocosos, situado en una terraza litoral intermedia a $15 \mathrm{~m} . \mathrm{snm}$. Las ocupaciones identificadas en este asentamiento han sido datadas por $\mathrm{C}^{14}$ en $5150 \pm 40$ AP (capa 2 inferior) y $4840 \pm 70 \mathrm{AP}$ (capa 2 superior), lo que permite asignar estos eventos a momentos transi- cionales entre los períodos Arcaico Medio y Arcaico Tardío (Castelleti et al. 2004). Gran cantidad de restos líticos, tanto derivados como instrumentos de acabada terminación, fueron recuperados desde el sector central del sitio, además de un entierro humano secundario asociado a una hoja bifacial lanceolada elaborada en calcedonia blanca (Castelleti et al. 2004). Este tipo de instrumentos rara vez se registra en asociación contextual, por lo que su hallazgo permite situar temporalmente su presencia en los registros costeros. Los tipos de puntas de proyectil pedunculadas registrados son similares a los recuperados en la parte inferior de la secuencia estratigráfica del conchal de Punta Morada (Bird 1988 [1943]) y en otros sitios de la costa norte entre Guanaqueros y Taltal (Schiappacasse y Niemeyer 1968, 1986; Fuentes et al. 2010). Estas puntas pedunculadas no se corresponden, sin embargo, con aquellas piezas semejantes (mas 
Patricio Galarce C., Gabriela Santander H.

\begin{tabular}{|c|c|c|c|c|}
\hline Componente ocupacional & Derivados & Instrumentos & Total restos & Proporción derivados a instrumentos \\
\hline \multicolumn{5}{|l|}{ Sector 1} \\
\hline Post $4840 \pm 70$ & 188 & 6 & 194 & 31.33 \\
\hline $4840 \pm 70$ & 157 & 6 & 163 & 26.17 \\
\hline $5150 \pm 40$ & 278 & 4 & 282 & 69.50 \\
\hline Total Sector 1 & 623 & 16 & 639 & 38.94 \\
\hline \multicolumn{5}{|l|}{ Sector 2} \\
\hline Post $4840 \pm 70$ & 168 & 5 & 173 & 33.60 \\
\hline $4840 \pm 70$ & 579 & 15 & 594 & 38.60 \\
\hline $5150 \pm 40$ & 4229 & 92 & 4321 & 45.97 \\
\hline Total Sector 2 & 4976 & 112 & 5088 & 44.43 \\
\hline Total Sectores 1 y 2 & 5599 & 128 & 5727 & 43.74 \\
\hline
\end{tabular}

Figura 2. Cuantificación de restos líticos por componente ocupacional en sitio 228/230.

no iguales) del Complejo Huentelauquén, que presentan dataciones muy anteriores a las que se registran para este contexto lítico específico.

Análisis distribucional de las frecuencias de restos líticos. El análisis frecuencial de restos líticos (Figura 2), muestra que es el sector 2 donde se observa una mayor concentración en la depositación lítica en el asentamiento. La marcada disparidad en la cantidad de restos depositados entre ambos sectores sugiere, en términos generales, que existieron diferencias claras entre áreas de actividad lítica operando en el lugar, lo que implicaría algún grado de diferenciación espacial en la organización de las referidas actividades.

Tan importante como la evaluación espacial resulta también ver si las diferencias gruesas que se observan entre ambos sectores del sitio se corresponden con variaciones relacionadas con los distintos momentos de ocupación. $\mathrm{Al}$ respecto, vemos que ambos sectores comparten como denominador común, que la mayor depositación de restos ocurrió en el primer componente ocupacional $(5150 \pm 40$ $\mathrm{AP})$, lo que sugiere que en ese momento se desarrolló una intensa actividad de talla lítica en el asentamiento. Por su parte, el segundo componente (4840 $\pm 70 \mathrm{AP})$ presenta una menor intensidad de trabajo lítico, puesto que la cantidad de desechos depositados es mucho más baja que en las ocupaciones precedentes.

El tercer componente del sitio (post $4840 \pm 70 \mathrm{AP}$ ), marca la última ocupación del lugar por grupos cazadores recolectores arcaicos. La reducida cantidad de materiales líticos depositados durante estos últimos momentos, indica un menor desarrollo de trabajo lítico, asociado a ocupaciones breves o esporádicas del lugar.

Otra aproximación a la cuantificación de restos, apunta a la relación entre el número de instrumentos vs. cantidades de derivados registrados. Este análisis es útil para ponderar el balance entre actividades de producción y de utilización lítica dentro de un conjunto (Shott 1993; Andrefsky 1998). Evidentemente, se pueden manejar varias relaciones comparativas, siendo aquí utilizada la proporción de derivados líticos respecto a instrumentos, para evaluar la posición relativa del conjunto en el proceso de manufactura y utilización de herramientas.

Como se aprecia para ambos sectores del sitio, encontramos que el componente más temprano es el que presenta mayor proporción de derivados líticos respecto a instrumentos (entre 69.50 y $45.97 \%$ ) que las ocupaciones posteriores del lugar. Ello sugiere que durante los primeros momentos, si bien se registra una alta cantidad de instrumentos ( $75 \%$ del total para el sitio) tenemos asociada una importante depositación de derivados provenientes de su manufactura, que indica que este sitio operó fuertemente como punto de elaboración de instrumentos para ser utilizados en el lugar o en sectores aledaños. Para los restantes componentes registrados, existe una menor proporción de derivados a instrumentos líticos que indica que las actividades de manufactura pierden importancia en detrimento de las actividades involucradas con la utilización de instrumentos y/o que existe proporcio- 
Sitio $228 / 230$ - Sector $1(5150 \pm 40 \mathrm{AP})$

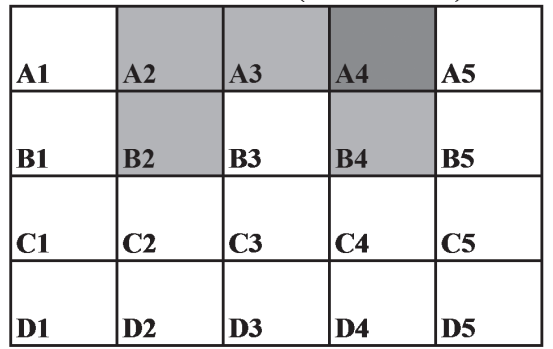

1 m

Sitio $228 / 230$ - Sector 1 (post $4840 \pm 70 \mathrm{AP}$ )

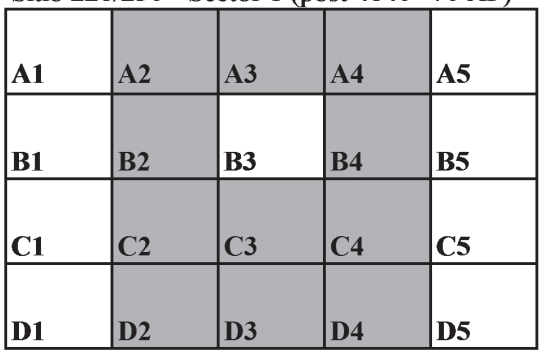

Sitio $228 / 230$ - Sector $1(4840 \pm 70$ AP $)$
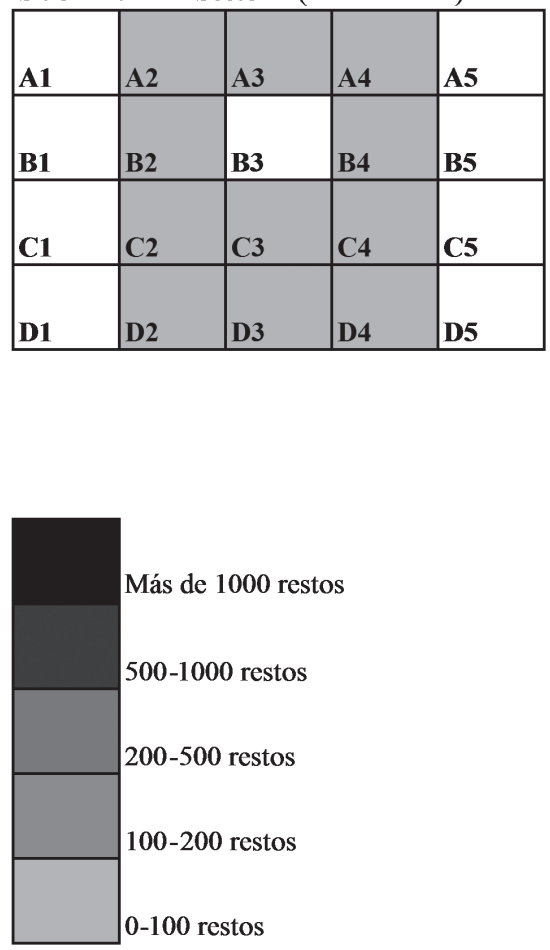

Figura 3. Distribución espacial de restos líticos por componente en sitio 228/230, Sector 1.

nalmente mayor proporción de descarte de instrumentos respecto a su manufactura en el lugar.

Dadas las diferencias espaciales y temporales identificadas, hay que tratar con más detalle este problema. Para esto se evaluó la distribución espacial de derivados entre las unidades excavadas en cada sector, con el objeto de identificar áreas diferenciales de depositación lítica.

El análisis distribucional realizado para este efecto, muestra que en el sitio se registran comportamientos disímiles tanto entre los sectores del mismo como entre los componentes ocupacionales definidos. Comencemos por el Sector 1 (Figura 3).

La distribución de derivados líticos en las unidades del sector 1 , muestra una primera situación que ocurre du- rante la ocupación de $5150 \pm 40 \mathrm{AP}$, donde vemos un área de actividad restringida con una clara concentración de materiales en la unidad A4 y una dispersión máxima asociada no mayor a $4 \mathrm{~m}^{2}$, configurando un locus de talla acotado, en este caso asociado a un afloramiento rocoso de gran tamaño. Posteriormente existe una segunda situación en la que tanto en el componente de 4840 70 AP como en el que le sigue, el área de actividad deja de conformar una agrupación definida, comportándose como un área de dispersión de restos más amplia, alcanzando una extensión aproximada de $12 \mathrm{~m}^{2}$. Estas importantes diferencias indican que este sector del asentamiento, con el tiempo, dejó de operar como un área de actividad de elaboración de instrumentos, para transformarse en un lugar de descarte marginal dentro del sitio, donde se realizaban actividades de talla no necesariamente relacionadas entre sí. 


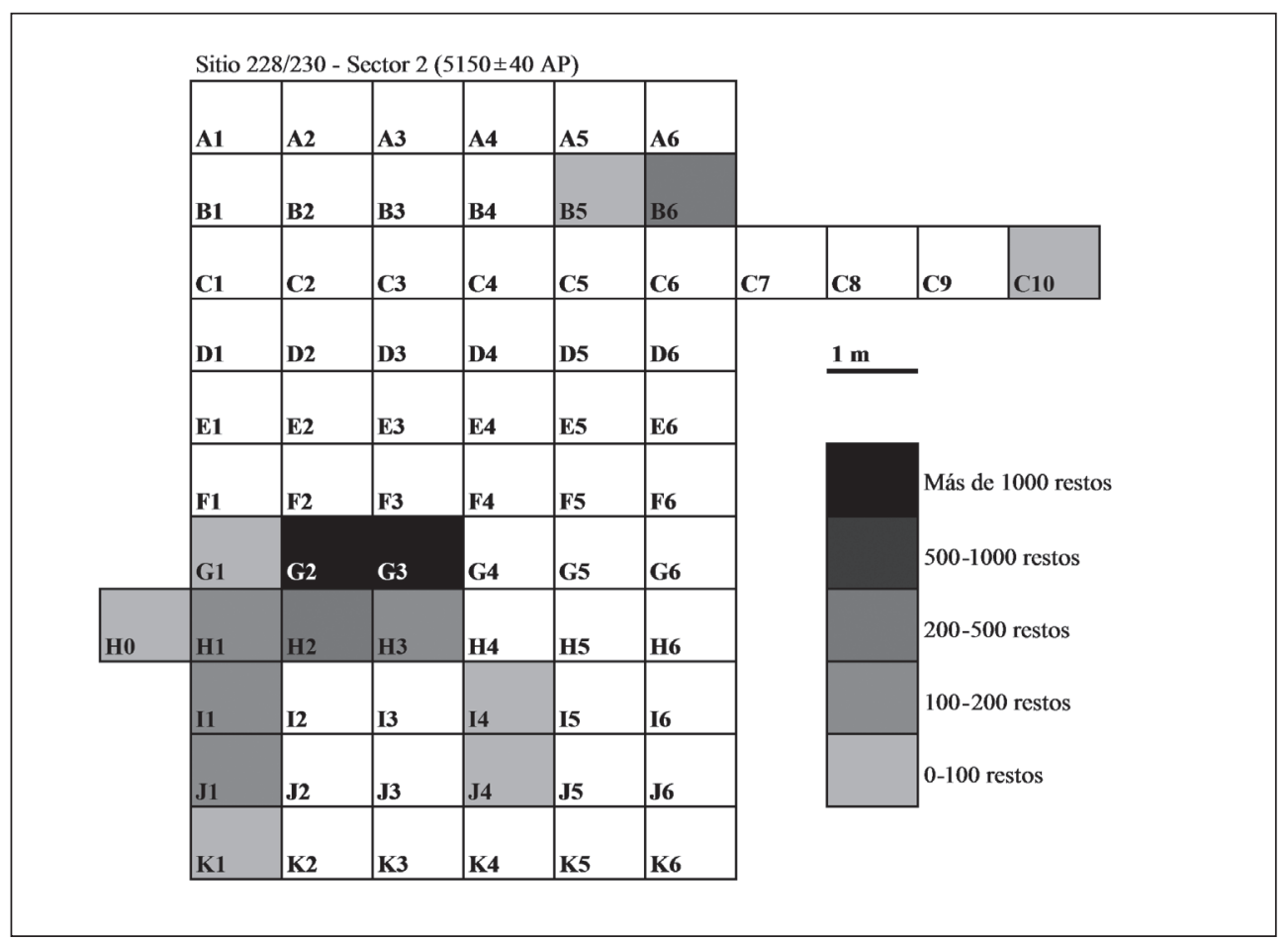

Figura 4. Distribución espacial de restos líticos en sitio 228/230, Sector 2, Componente 5150 4 O AP.

La situación del Sector 2 muestra mayor complejidad en la deposición de derivados líticos. De este modo, el componente ocupacional de 5150 $440 \mathrm{AP}$ (Figura 4), permite apreciar la existencia de un locus de talla con presencia masiva de materiales en el punto de mayor concentración (unidades $\mathrm{G}_{2}$ y G3). A partir de éste observamos que las frecuencias disminuyen progresivamente pasando de cantidades medias a bajas de material, conformando un patrón de dispersión radial de restos, donde un bloque rocoso situado en el lugar de las unidades I2, I3, J2 y J3 estructura la forma que adquiere dicha área de dispersión. Este locus se ubica en la parte central del asentamiento, donde se realizaron las actividades de talla más intensas en el lugar. Al otro lado del bloque se registró un entierro secundario, con presencia de dos instrumentos líticos como parte de las ofrendas, incluyendo una hoja bifacial en calcedonia blanca, mostrando que esta área se organiza de un modo más complejo que la mera producción de instrumentos. Un segundo locus se denota en las unidades $\mathrm{B} 6$ y $\mathrm{B} 5$, donde otro afloramiento de roca favoreció la realización de marcadas actividades de producción lítica en ese lugar.
La distribución de restos líticos en este sector, configurando varios loci de talla emplazados en los afloramientos rocosos del lugar, sumado a la presencia de rasgos con funcionalidad especial, señala a nuestro juicio el área residencial del asentamiento.

Para el componente de $4840 \pm 70$ AP (Figura 5), observamos la desaparición de los loci identificados en el momento anterior y su reemplazo por áreas discretas y poco intensas de trabajo lítico. Aunque se mantienen dos concentraciones principales en el Sector 2, estas son menos masivas en términos de las cantidades de materiales depositados así como en su desarrollo horizontal; sin embargo, se mantiene como conducta la asociación estrecha entre loci de talla y afloramientos rocosos. En resumen, la distribución de restos líticos para el componente de $4840 \pm 70 \mathrm{AP}$, tanto en el Sector 1 como en el 2, indica que en el sitio hubo un menor énfasis en la producción de cantidades significativas de instrumentos.

Por último, tenemos la situación que nos muestra el componente post $4840 \pm 70 \mathrm{AP}$ (Figura 6 ), donde apreciamos 


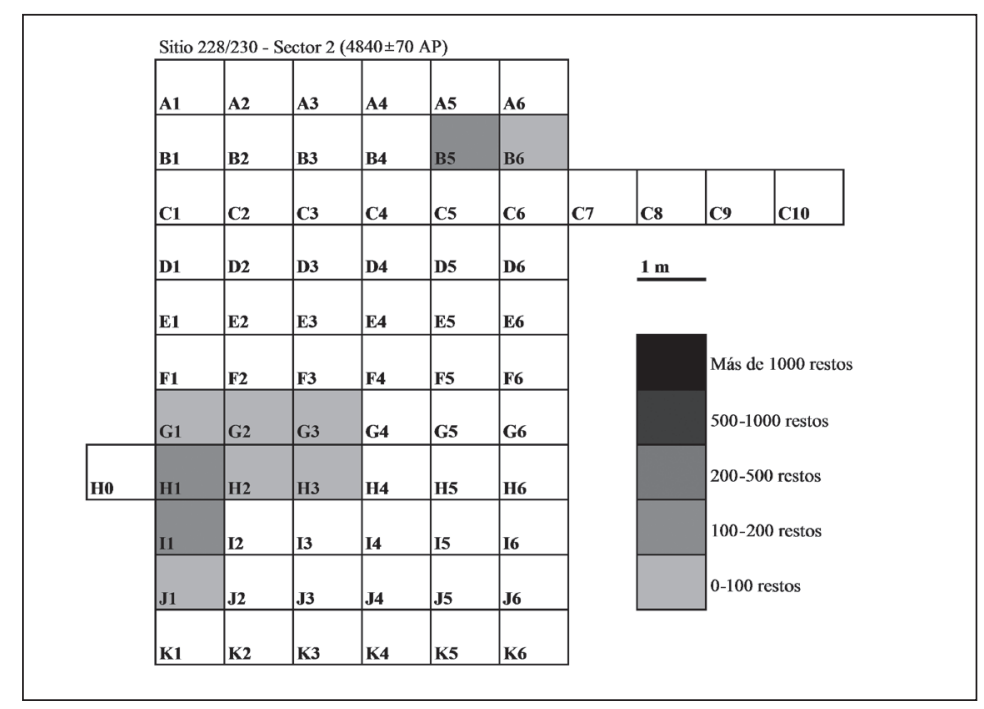

Figura 5. Distribución espacial de restos líticos en sitio 228/230, Sector 2, Componente 4840 770 AP.

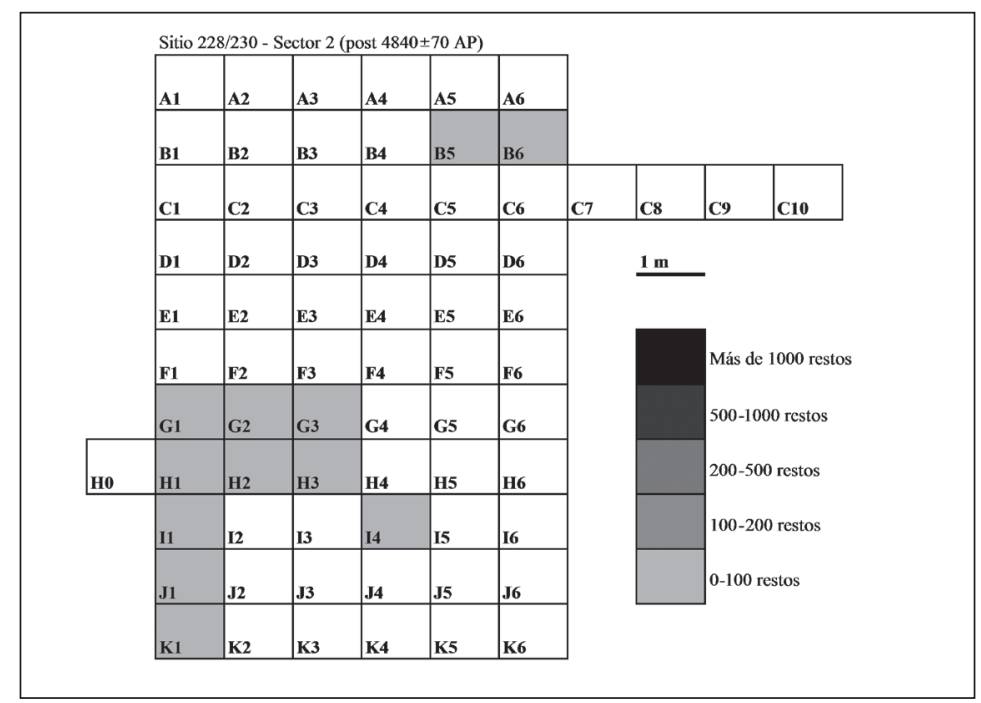

Figura 6. Distribución espacial de restos líticos en sitio 228/230, Sector 2, Componente post 4840 \pm 70 AP.

la distribución de derivados líticos en forma dispersa, sin un patrón de concentración aparente. Inferimos para el sitio una ocupación de características efímeras con escasas actividades de talla lítica representadas en este componente.

En síntesis, es posible apreciar la existencia de un primer momento de ocupación, de carácter residencial, donde el desarrollo de las actividades de talla lítica muestra la presencia de áreas o loci de trabajo espacialmente segregados y marcados por la depositación de grandes cantidades de restos líticos, asociados a una fuerte actividad de manufactura de instrumentos. Las ocupaciones posteriores se caracterizaron por una organización simplificada del espacio, en un cuadro marcado por un trabajo lítico menos intenso y un carácter menos residencial. 


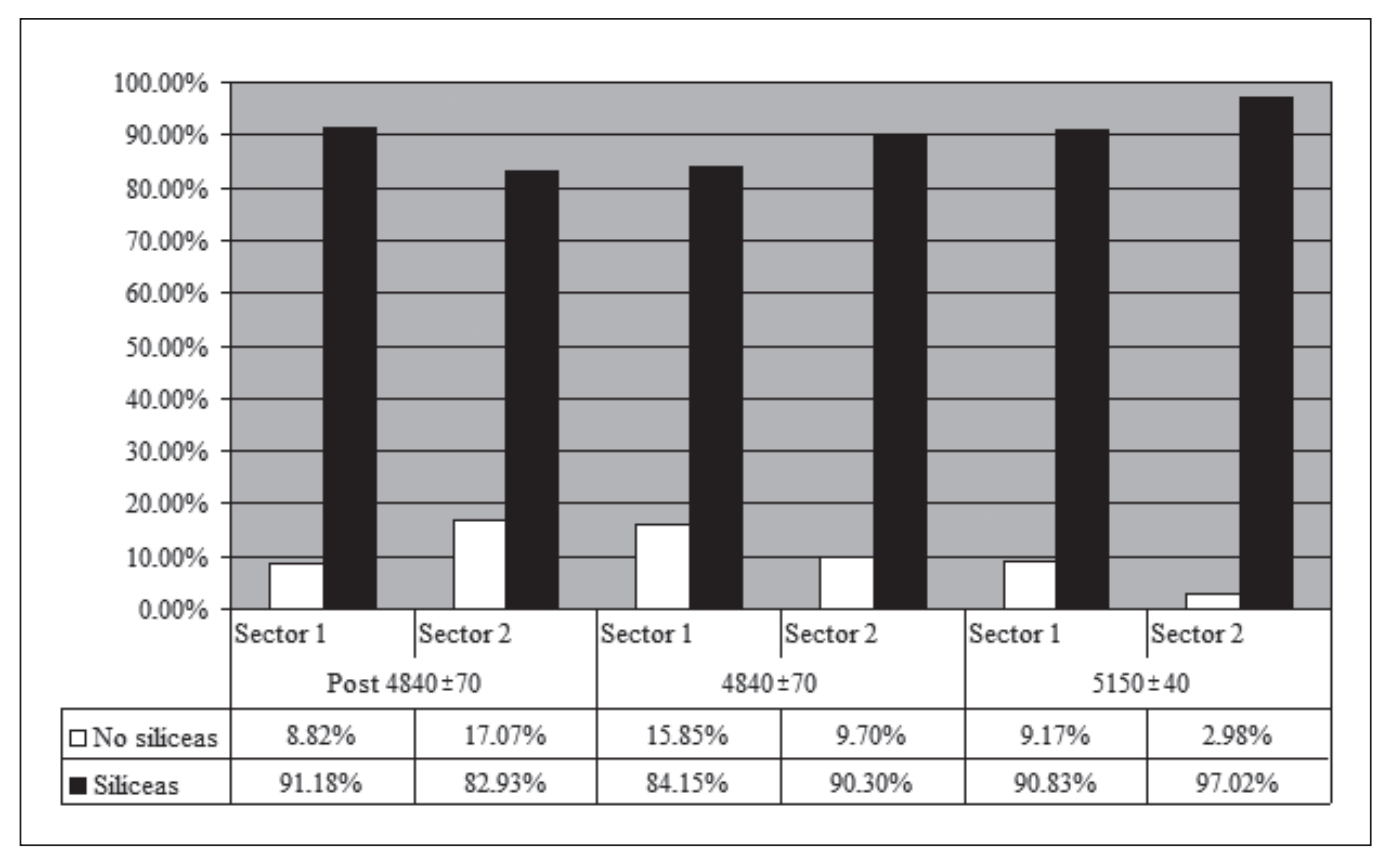

Figura 7. Frecuencia de materias primas por componente ocupacional y sector en sitio 228/230.

Análisis tecnológico de materias primas y derivados líticos. Un primer aspecto a considerar dentro de la discusión del problema tecnológico se refiere a aquellos materiales que son objeto de modificaciones durante el trabajo lítico, es decir, las materias primas. Conocer su comportamiento dentro de un determinado conjunto lítico permite acceder a problemas tan importantes como las estrategias reductivas desempeñadas por un grupo de talladores y su relación con conductas de aprovisionamiento lítico y de implementación de una tecnología basada en la utilización de la piedra (Whittaker 1994; Andrefsky 1998). Para el caso de los sitios de Taltal, existe un denominador común marcado por la utilización extensiva de materias primas silíceas y silicificadas de alta calidad, recursos que parecen haber sido abundantes y accesibles a partir de áreas cercanas a la costa. Con esta idea en mente, revisemos lo que ocurre en el sitio $228 / 230$.

El porcentaje de derivados analizados tecnológicamente y por materias primas es de $83.43 \%$ (4662 de 5599 piezas). Como se puede apreciar en la Figura 7 , un porcentaje altamente mayoritario del material lítico corresponde a materias primas silíceas de alta calidad para la talla.
Las rocas silíceas y silicificadas de alta calidad, fueron los recursos que sirvieron como soporte tecnológico de la industria lítica del sitio, y son factibles de encontrar en el área interior aledaña a la costa de Taltal, principalmente en las planicies de pampas que se ubican al oriente del macizo de la cordillera costera (Núñez 1984; Peralta et al. 2010). El espectro de rocas silíceas registradas en el sitio incluye diversos tipos de calcedonias, jaspes y cherts presentes en variados colores y texturas. De acuerdo con sus atributos tecnológicos (relación grano/fractura), estas rocas pueden ser catalogadas como recursos líticos de alta calidad para la talla (Luedtke 1979, 1992; Semenov 1981; Aragón y Franco 1997; Andrefsky 1998). Las materias primas no silíceas registradas corresponden a una serie de rocas andesíticas y basálticas de calidad media a baja para la talla, las cuales parecen estar disponibles en sectores cercanos al asentamiento, como podría ser el caso de algunas playas de guijarros o depósitos de acarreo presentes en las quebradas del área costera (Castelleti com. pers. 2004).

El problema de las materias primas líticas obtenidas y procesadas por los ocupantes del sitio parece ser de fácil resolución, con una situación marcada por la utilización 


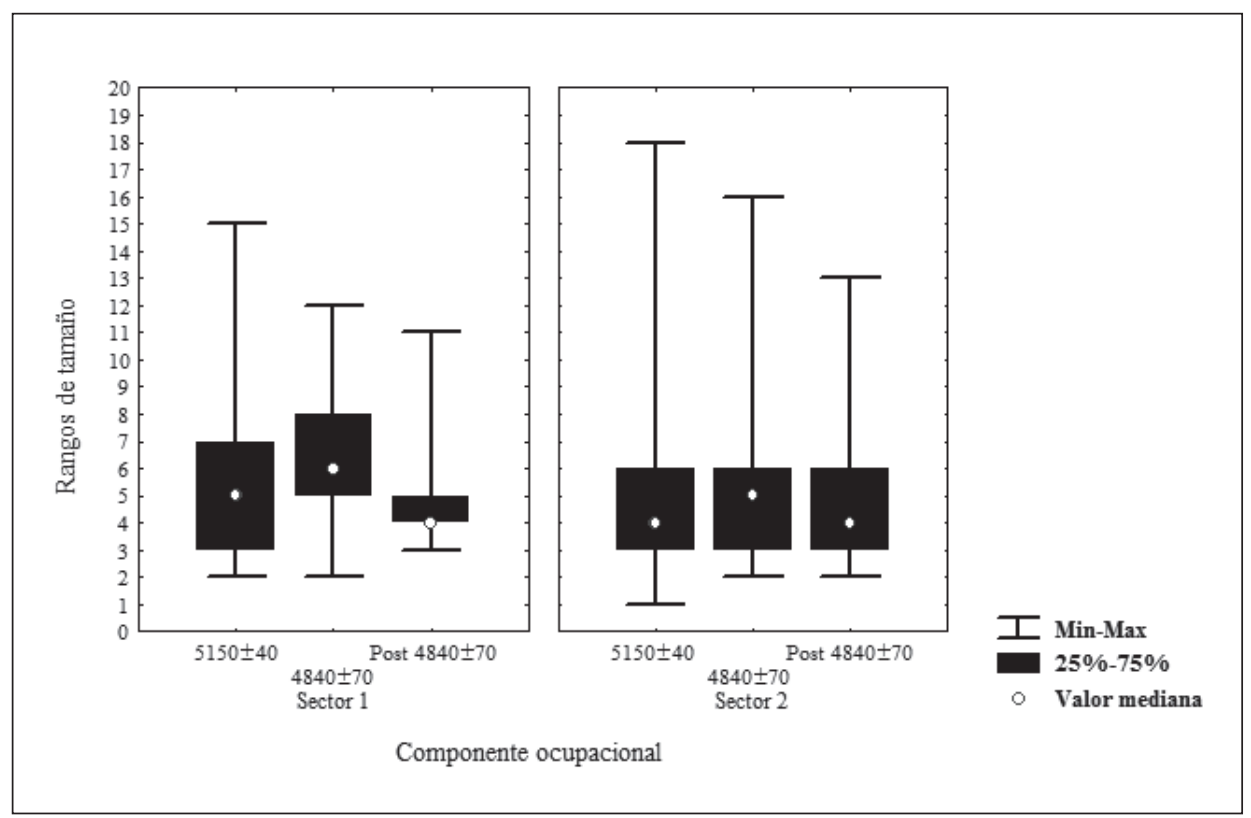

Figura 8. Distribución de rangos de tamaños en derivados por sector y componente ocupacional en sitio 228/230.

mayoritaria de recursos líticos silíceos y silicificados de alta calidad. La variabilidad apreciable en términos de colores, texturas y otros atributos propiamente tecnológicos puede ser atribuida a factores internos de presentación de los recursos en fuentes de aprovisionamiento heterogéneas, más que a imperativos derivados de la disponibilidad o acceso a las mismas (Luedtke 1979, 1992). De este modo, la discusión que sigue respecto a las características mostradas por el procesamiento de las materias primas, se abocará a evaluar la situación de los recursos líticos como un conjunto, sin recalcar especialmente las diferencias internas de los distintos tipos de rocas entre sí.

La variación morfométrica de los derivados líticos arroja luces respecto a las características que presenta el desarrollo del proceso reductivo enfocado a la elaboración de instrumentos tallados (Sullivan y Rozen 1985; Andrefsky 1998, 2001, 2008; Bradbury y Carr 1999). Tres variables morfométricas serán evaluadas, correspondientes al tamaño general de la pieza, la morfología de la plataforma y la variación métrica de la misma.

El primer indicador evaluado corresponde al tamaño general de la pieza, para lo cual se empleó un método por rangos máximos de tamaño a partir de círculos con diámetro creciente cada 5 mm (Andrefsky 1998; De Souza 2003; Galarce 2004), con el cual se puede estimar el eje máximo de la pieza, en el sentido del largo y ancho. Los resultados para el conjunto de 228/230 por sector y componente ocupacional muestran situaciones interesantes (Figura 8). Siguiendo la lógica indicada por la distribución de restos líticos en ambos sectores del asentamiento, vemos que el sector 2 presenta una estructura de tamaños para el componente de $5150 \pm 40$ $\mathrm{AP}$, con una situación marcada por intensas actividades de talla lítica que desarrolla un proceso reductivo completo. Sin embargo, la notoria concentración de tamaños en rangos bajos desde 03 a $07(15-35 \mathrm{~mm})$, tiende a indicar una mayor importancia de momentos reductivos avanzados, como la manufactura de instrumentos altamente formatizados. Similar evaluación se puede hacer de lo que ocurre en el mismo componente del Sector 1 . Al respecto podemos relacionar el alto grado de concentración espacial de las actividades de producción con la importancia mayoritaria de la manufactura de instrumentos.

Para los componentes ocupacionales posteriores, la distribución de tamaños indica mayor importancia relativa de momentos de trabajo relacionados con la elaboración 


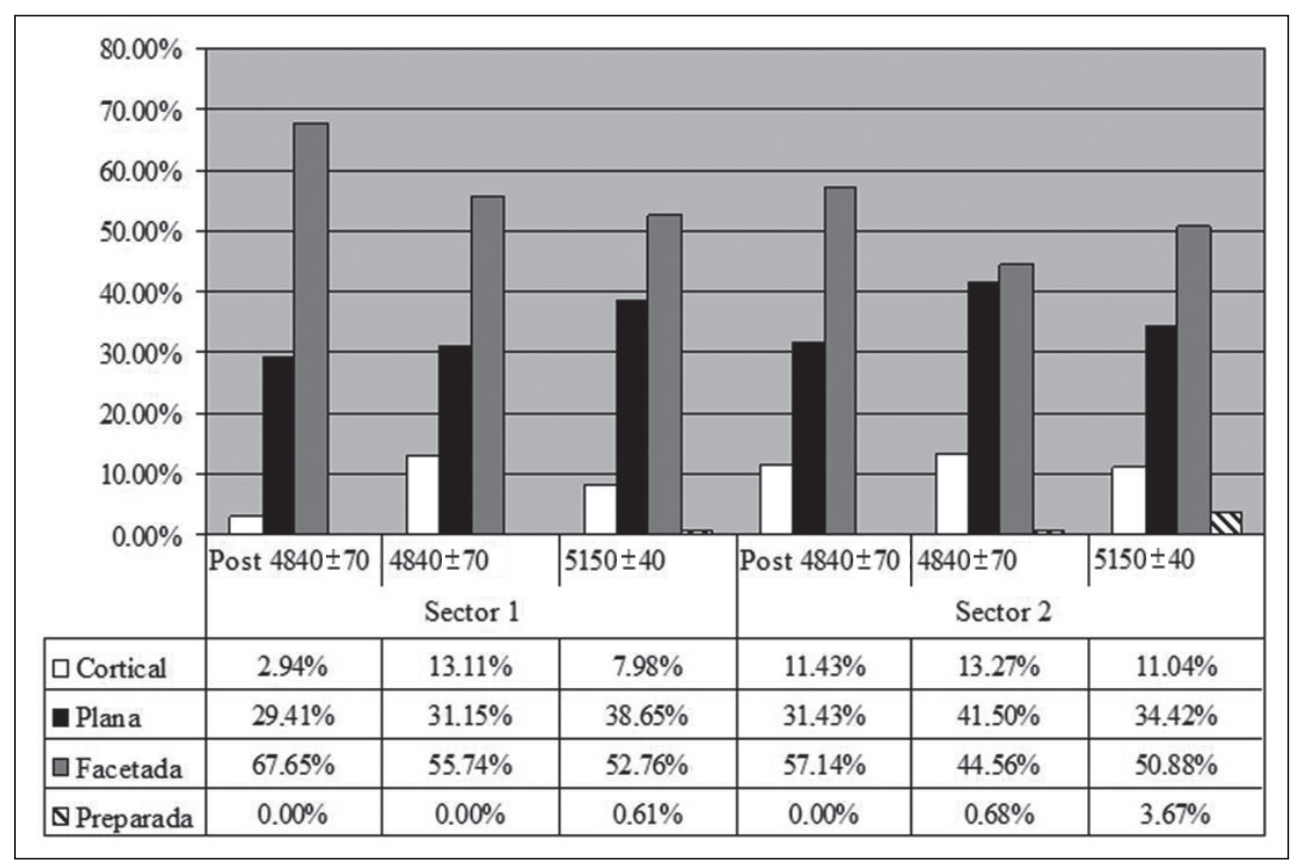

Figura 9. Frecuencia de morfologías de plataformas por sector y componente en sitio 228/230.

de instrumentos. La menor amplitud de valores de tamaño en estos conjuntos más tardíos, sugiere que, si bien las secuencias de talla parecen reflejar la presencia de desbaste de núcleos, esto ocurre en menor medida que en las primeras ocupaciones del lugar. Estas situaciones reflejarían también el carácter menos residencial de la ocupación del sitio en los dos últimos componentes.

Dado que la manufactura de instrumentos se concentra en loci específicos y discretos, vale la pena indagar sobre la clase de herramientas producidas en el lugar durante su ocupación. Un buen indicador al respecto se refiere a las variaciones morfológicas y métricas de la plataforma de percusión o presión, porción del derivado lítico que experimenta una serie de cambios físicos durante el proceso reductivo de materia prima (Whittaker 1994; Andrefsky 1998; Galarce 2004).

El primer indicador de variación de la plataforma es morfológico y se refiere a la trayectoria ideal de cambio que se da durante las actividades de talla, desde momentos iniciales a avanzados, en la morfología de las plataformas de percusión. En nuestro esquema, la presencia de plataformas corticales es un indicador fuerte de momentos iniciales de talla (desbaste de núcleos), mientras que la generación progresiva y creciente de plataformas facetadas y preparadas es un indicador inequívoco de la reducción bifacial de matrices (Cornejo y Galarce 2010). La situación de las plataformas planas es más confusa, puesto que su aparición se produce tanto durante el desbaste de núcleos como durante la reducción de matrices. Planteado el problema de este modo veamos qué sucede en el contexto lítico de 228-230 (Figura 9).

Para estos conjuntos resulta evidente el claro predominio de plataformas facetadas y planas sobre el resto de los tipos morfológicos, indicando el importante papel que cumplió la reducción bifacial completa de matrices, mientras que la presencia de plataformas corticales indican el ingreso y trabajo de matrices provenientes de núcleos, que se encuentran en estados iniciales de reducción bifacial (Galarce 2005).

La evaluación temporal de las variaciones en la forma de las plataformas, muestra una tendencia desde situaciones caracterizadas por el desarrollo de secuencias reductivas bifaciales completas ( $5150 \pm 40 \mathrm{AP}$ y $4840 \pm 70 \mathrm{AP})$, hacia una situación más tardía donde un mayor desbalance a 


\begin{tabular}{|l|c|c|c|c|}
\hline $\begin{array}{l}\text { Categoría } \\
\text { morfofuncional }\end{array}$ & Post 4840 $\mathbf{7 0}$ & $\mathbf{4 8 4 0 \pm 7 0}$ & $\mathbf{5 1 5 0 \pm 4 0}$ & Total \\
\hline Puntas proyectil & $2(33.33 \%)$ & $1(16.67 \%)$ & $0(0.0 \%)$ & $3(18.75 \%)$ \\
\hline Preformas & $1(16.67 \%)$ & $4(66.67 \%)$ & $3(75.0 \%)$ & $8(50.0 \%)$ \\
\hline $\begin{array}{l}\text { Instrumentos } \\
\text { lasca bifacial }\end{array}$ & $1(16.67 \%)$ & $0(0.0 \%)$ & $1(25.0 \%)$ & $2(12.50 \%)$ \\
\hline Cuchillos & $1(16.67 \%)$ & $\circ(0.0 \%)$ & $\circ(0.0 \%)$ & $1(6.25 \%)$ \\
\hline $\begin{array}{l}\text { Denticulados } \\
\text { o muescas }\end{array}$ & $1(16.67 \%)$ & $0(0.0 \%)$ & $\circ(0.0 \%)$ & $1(6.25 \%)$ \\
\hline $\begin{array}{l}\text { Raspadores } \\
\text { dorso alto }\end{array}$ & $0(0.0 \%)$ & $1(16.67 \%)$ & $0(0.0 \%)$ & $1(6.25 \%)$ \\
\hline Total & $6(100 \%)$ & $6(100 \%)$ & $4(100 \%)$ & $16(100 \%)$ \\
\hline
\end{tabular}

Figura 10. Frecuencia de categorías morfofuncionales en sitio 228/230, Sector 1.

favor de las plataformas facetadas sugiere secuencias de talla enfocadas en el desbaste más avanzado de las matrices bifaciales (post $4840 \pm 70 \mathrm{AP}$ ). Es posible relacionar esta variación tecnológica temporal, con un cambio en el carácter espacial y funcional de la ocupación, desde un contexto de uso más generalizado a uno más específico.

Análisis morfofuncional de los instrumentos líticos. La representación de clases de instrumentos líticos descartados en el sitio permiten contrastar algunas de las interpretaciones esbozadas respecto al comportamiento y orientación de los conjuntos líticos del sitio. Abordamos las diferentes clases de instrumentos solo desde una perspectiva morfofuncional, prestando especial atención a los atributos morfológicos y tecnológicos más relacionados a características funcionales de los instrumentos (Bate 1971; Piel-Desruisseaux 1989; Jackson 1991). En este sentido, una aproximación morfofuncional permite una buena descripción de las maneras como los talladores prehistóricos manufacturaron las herramientas que utilizaron para desempeñar las actividades desarrolladas dentro de los distintos asentamientos.

De todos modos, reconocemos la necesidad de implementar metodologías de aproximación más completas y seguras al problema funcional de las herramientas líticas, como sería el caso de los análisis de huellas de uso en bajos y altos aumentos (Semenov 1981; Mansur 1997).

Para el Sector 1 del sitio (Figura 10), vemos que las frecuencias de categorías morfofuncionales muestran una reducida cantidad de instrumentos descartados, a la vez que estos representan una baja diversidad de actividades productivas líticas desarrolladas en este sector.

La reducida cantidad de instrumentos registrados en este sector atenta contra una evaluación significativa de las actividades de uso/descarte lítico que tuvieron lugar. Sin embargo, se aprecia una mayor frecuencia para las categorías manufacturadas a partir de matrices bifaciales (puntas de proyectil, preformas, cuchillos e instrumentos sobre lascas de desbaste bifacial). Esta situación es coherente con la información distribucional y tecnológica de los derivados líticos, que definen este sector como un área de actividad discreta, con gran predominio de actividades de talla bifacial, debido tanto al carácter circunscrito del locus de talla como a la significativa frecuencia de plataformas con morfologías planas y facetadas. La representación de instrumentos sugiere que relativamente pocas actividades de elaboración, utilización y descarte lítico tuvieron lugar en esta parte del asentamiento. Una explicación alternativa a esta situación podría vincularse con la salida de instrumentos desde el sitio para ser utilizados o descartados en otros lugares del área.

Distinto es el caso del Sector 2, donde se aprecia una mayor diversidad de categorías morfofuncionales representadas, así como una mayor cantidad de instrumentos depositados en esta parte del sitio. La situación aquí visualizada nos indica que hubo una alta diversidad de actividades de elaboración y utilización de herramientas 
Patricio Galarce C., Gabriela Santander H.

\begin{tabular}{|c|c|c|c|c|}
\hline $\begin{array}{l}\text { Categoría } \\
\text { morfofuncional }\end{array}$ & Post $4840 \pm 70$ & $4840 \pm 70$ & $5150 \pm 40$ & Total \\
\hline Puntas proyectil & $0(0.0 \%)$ & $3(20.0 \%)$ & $26(28.26 \%)$ & $29(25.89 \%)$ \\
\hline Bifaces & $2(40.0 \%)$ & $4(26.67 \%)$ & $30(32.61 \%)$ & $36(32.14 \%)$ \\
\hline $\begin{array}{l}\text { Instrumentos } \\
\text { lasca bifacial }\end{array}$ & $0(0.0 \%)$ & $0(0.0 \%)$ & $4(4 \cdot 35 \%)$ & $4(3.57)$ \\
\hline Cuchillos & $2(40.0 \%)$ & $3(20.0 \%)$ & $10(10.87 \%)$ & $15(13.39 \%)$ \\
\hline $\begin{array}{l}\text { Denticulados } \\
\text { o muescas }\end{array}$ & $0(0.0 \%)$ & $1(6.67 \%)$ & $13(14.13 \%)$ & $14(12.50 \%)$ \\
\hline Leznas & $0(0.0 \%)$ & $0(0.0 \%)$ & $1(1.09 \%)$ & $1(0.89 \%)$ \\
\hline $\begin{array}{l}\text { Raspadores } \\
\text { dorso alto }\end{array}$ & $0(0.0 \%)$ & $2(13.33 \%)$ & $4(4 \cdot 35 \%)$ & $6(5.36 \%)$ \\
\hline Cepillos & $0(0.0 \%)$ & $0(0.0 \%)$ & $1(1.09 \%)$ & $1(0.89 \%)$ \\
\hline Tajadores & $0(0.0 \%)$ & $0(0.0 \%)$ & $1(1.09 \%)$ & $1(0.89 \%)$ \\
\hline Núcleos & $1(20.0 \%)$ & $2(13.33 \%)$ & $2(2.17 \%)$ & $5(4.46 \%)$ \\
\hline Total & $5(100 \%)$ & $15(100 \%)$ & $92(100 \%)$ & $112(100 \%)$ \\
\hline
\end{tabular}

Figura 11. Frecuencia de categorías morfofuncionales por componente ocupacional en sitio 228/230, Sector 2.

representadas en las áreas de actividad identificadas, así como una significativa frecuencia de piezas descartadas (Figura 11).

En el Sector 2 se concentran las principales áreas con actividades de talla, uso y descarte lítico dentro del sitio, destacando la alta proporción que alcanzan las categorías morfofuncionales de instrumentos formatizados de manera bifacial durante la ocupación del sitio, aunque existe una importante frecuencia en el componente inicial (5150 $\pm 40 \mathrm{AP})$ para los instrumentos con borde denticulado o en muesca, que pueden ser utilizados en actividades relacionadas con la presencia de puntas de proyectil y otros bifaces, como la preparación y acondicionamiento de enmangues y astiles. La orientación funcional de los conjuntos instrumentales en el sitio apunta a un claro predominio en la manufactura y utilización de instrumentos bifaciales sobre otras categorías morfofuncionales de herramientas. Esta situación confirma el cuadro esbozado respecto a la importancia que alcanza la manufactura bifacial en los distintos loci de talla presentes en el sector, al mismo tiempo que la mayor diversidad de actividades líticas registradas indica un carácter más residencial para esta parte del asentamiento, especialmente para el componente más temprano identificado.

De acuerdo con los resultados del análisis morfofuncional realizado, existe un marcado paralelismo tecnológico y funcional entre los conjuntos de derivados y los de instrumentos líticos. Ambas perspectivas de aproximación tienden a indicar que el asentamiento funcionó de manera similar a un campamento residencial de tareas específicas, en el cual las actividades líticas más importantes tuvieron que ver con la reducción de matrices bifaciales. La relativa importancia que se registra para categorías involucradas con la realización de actividades domésticas, como los instrumentos de bordes denticulados o en muesca, para las primeras ocupaciones, sugiere que su carácter residencial fue más marcado en dichos momentos que en tiempos posteriores. Podemos postular una trayectoria funcional y depositacional, donde los primeros eventos ocupacionales conforman loci de talla bien definidos y segregados, con alta diversidad artefactual y un carácter más residencial, sucedidos por ocupaciones donde el asentamiento deja de cumplir un rol altamente residencial y es reemplazado por uno específico, donde las actividades de reducción lítica, casi exclusivamente bifaciales, mostrarían una menor diversidad de actividades funcionales.

\section{Sitio 226-5}

Este sitio corresponde a un asentamiento asociado a un alero rocoso, ubicado en una terraza marina alta, en la ladera norte del portezuelo de la punta de Caleta Oliva, unos $15 \mathrm{~km}$ al norte de Taltal, donde se han obtenido algunas 


\begin{tabular}{|l|r|r|r|r|}
\hline Componente ocupacional & Derivados & Instrumentos & Total restos & $\begin{array}{r}\text { Proporción derivados a } \\
\text { instrumentos }\end{array}$ \\
\hline No determinado & 633 & 62 & 695 & 10.21 \\
\hline Post $10.040 \pm 60$ & 1316 & 25 & 1341 & 52.64 \\
\hline $10.040 \pm 60$ & 1394 & 48 & 1442 & 29.04 \\
\hline $10.290 \pm 60$ & 453 & 18 & 471 & 25.17 \\
\hline Total sitio & 3796 & 153 & 3949 & 24.81 \\
\hline
\end{tabular}

Figura 12. Cuantificación de restos líticos por componente ocupacional en sitio 226-5.

de las dataciones absolutas más tempranas para esta zona costera: de 10.290 \pm 60 AP y $10.040 \pm 60$ AP (Castelleti et al. 2010). Estas fechas se corresponden sincrónicamente con las ocupaciones arcaicas tempranas del Complejo Huentelauquén identificadas en otros sectores de la costa árida y semiárida, como la Chimba 13 en Antofagasta, El Obispo 1 en Chañaral, Huentelauquén en la desembocadura del Choapa y Punta Ñagué en Los Vilos (Llagostera 1979; Jackson 1993; Llagostera et al. 2000, Weisner et al. 2000; Jackson y Méndez 2005). Diversos indicadores artefactuales, permiten correlacionar ergológicamente las ocupaciones datadas del lugar con aquellas del mencionado complejo cultural, a saber, puntas triangulares o lanceoladas con pedúnculo ojival convergente, puntas triangulares de base recta, instrumentos multifuncionales sobre lascas de adelgazamiento bifacial y claras evidencias del trabajo reductivo de grandes matrices bifaciales.

Un componente ocupacional alfarero tardío fue también identificado, probablemente asociado a rasgos de inhumación disturbados por el trabajo de saqueadores (Castelleti et al. 2010). Los indicadores artefactuales más representativos de esta ocupación corresponden a puntas de proyectil triangulares de bases cóncavas y triangulares con aletas y pedúnculo restringido, de reducidas dimensiones.

Análisis distribucional de los restos líticos. Debido a la fuerte disturbación antrópica de los depósitos al interior del alero rocoso, las excavaciones arqueológicas fueron realizadas en el sector exterior del sitio, por lo que no tenemos la posibilidad de evaluar el comportamiento distribucional de los restos líticos en el interior. Las comparaciones, entonces, serán enfocadas principalmente entre los componentes ocupacionales definidos para este sector del sitio.

El análisis de cuantificación de restos líticos (derivados e instrumentos) muestra situaciones destacables (Figura 12).
Como se aprecia, el primer componente ocupacional del sitio (10.290 $\pm 60 \mathrm{AP})$, presenta una baja cantidad de desechos depositados en contraposición con los dos componentes ocupacionales que le siguen. Puesto que tanto el componente de $10.040 \pm 60 \mathrm{AP}$ como el que le sucede muestran una mayor actividad de trabajo lítico, representada por una mayor cantidad de restos, podemos sugerir que durante la primera ocupación, se registró una menor actividad de talla lítica, la cual se volvió más intensa en las ocupaciones posteriores.

Al considerar las proporciones existentes entre derivados e instrumentos líticos, notamos que tanto el componente de $10.290 \pm 60$ AP como el de $10.040 \pm 60$ $\mathrm{AP}$, se presentan como conjuntos con una baja proporción de derivados a instrumentos, lo cual tiende a sugerir que en esos momentos la actividad productiva contempló tanto la elaboración como la utilización de instrumentos, probablemente dentro de una lógica de ocupación de carácter residencial. Para la situación del componente post $10.040 \pm 60 \mathrm{AP}$, el notorio aumento de la actividad de elaboración lítica por sobre las de utilización, expresada en una mayor cantidad de derivados por instrumentos depositados, sugiere que el asentamiento funcionó dentro de una lógica menos residencial, donde una porción importante de los instrumentos pudieron haber sido transportados a otras locaciones para ser utilizados y posteriormente descartados.

De acuerdo con las situaciones marcadas por el análisis de cuantificaciones y proporciones, resulta conveniente evaluar qué ocurre en términos distribucionales con la actividad de talla lítica, con el objeto de calibrar algunas de las afirmaciones anteriores y también considerar nuevos aspectos referidos al comportamiento de los conjuntos líticos de este sitio. 


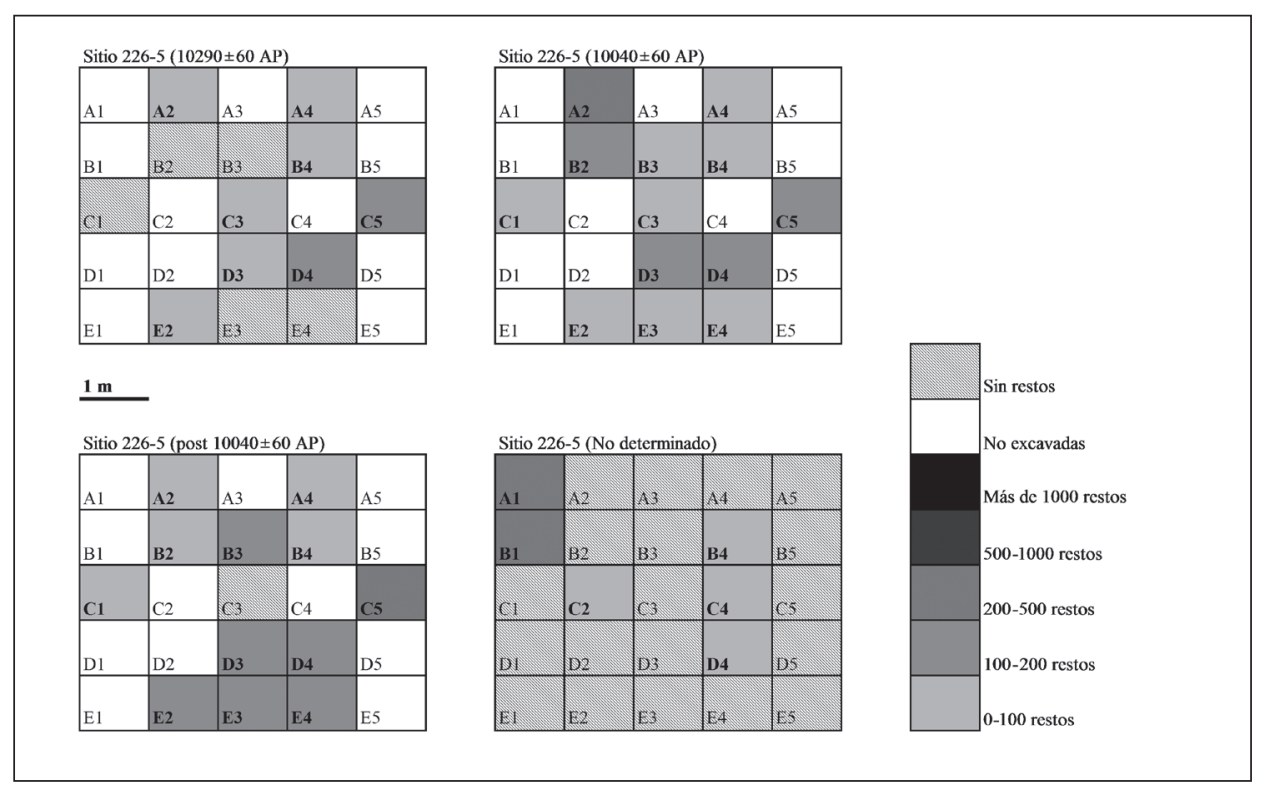

Figura 13. Distribución espacial de restos líticos por componente en sitio 226-5.

El comportamiento distribucional de restos líticos nos muestra un escenario marcado por fuertes variaciones espaciales en la disposición de las áreas de trabajo lítico dentro del sitio (Figura 13). De este modo, tenemos una primera situación (10.290 $\pm 60 \mathrm{AP})$ en la cual no se constituye todavía un locus de talla claramente definido y donde la distribución de restos se muestra en un patrón disperso. Las unidades con valores más altos ( $\mathrm{C}_{5}$ y $\left.\mathrm{D}_{4}\right)$, se ubican claramente en la parte más exterior del sitio, insinuando la presencia de un locus más concentrado en dichos sectores. La baja cantidad de restos y su carácter disperso nos indica que la ocupación del lugar pudo haber sido breve, con pocas actividades de talla lítica realizadas en el sitio.

Marcadas diferencias con la situación anterior se aprecian para el componente de $10.040 \pm 60 \mathrm{AP}$, donde vemos la configuración de un locus de talla definido en el sector más cercano al alero (unidades A2 y B2), con su correspondiente dispersión radial de restos, mientras que hacia el borde exterior del sitio (unidades $\mathrm{D}_{3}, \mathrm{D}_{4}$ y $\mathrm{C}_{5}$ ), encontramos otra concentración de talla menos densa, espacialmente coincidente con la distribución observada en el anterior componente. Este cuadro tiende a indicar un grado mayor de organización espacial de la actividad de talla lítica dentro del asentamiento con la conforma- ción de áreas de trabajo separadas y utilización más potente del espacio aledaño a la boca del alero. El carácter de la ocupación, aunque tal vez corto en duración, reflejaría también una importancia significativa de actividades más residenciales.

Otra situación se observa para el tercer y último componente ocupacional (post 10.040 $\pm 60 \mathrm{AP}$ ), donde existió un locus de talla bien definido y denso en el borde exterior del área excavada (unidad $\mathrm{C}_{5}$ ) asociado a un descenso en la intensidad de trabajo lítico cerca de la boca del alero, donde ya no se configura un locus de talla claramente definido. La ocupación podría tener un carácter más residencial, en términos de las actividades líticas que se desarrollaron en el lugar.

Por último, la distribución de restos en el componente no determinado nos indica el lugar (unidades $\mathrm{A} 1$ y $\mathrm{B}$ ) donde los depósitos fueron disturbados por saqueadores, quienes removieron por lo menos una tumba del Alfarero Tardío que se encontraba en la parte interior del alero. Evidentemente, esta disturbación del depósito afectó fuertemente las áreas de actividad lítica que pudieron encontrarse en esta parte del sitio. Hay que destacar, sin embargo, la gran cantidad de restos líticos que fueron recuperados del sector de cuadrículas distur- 


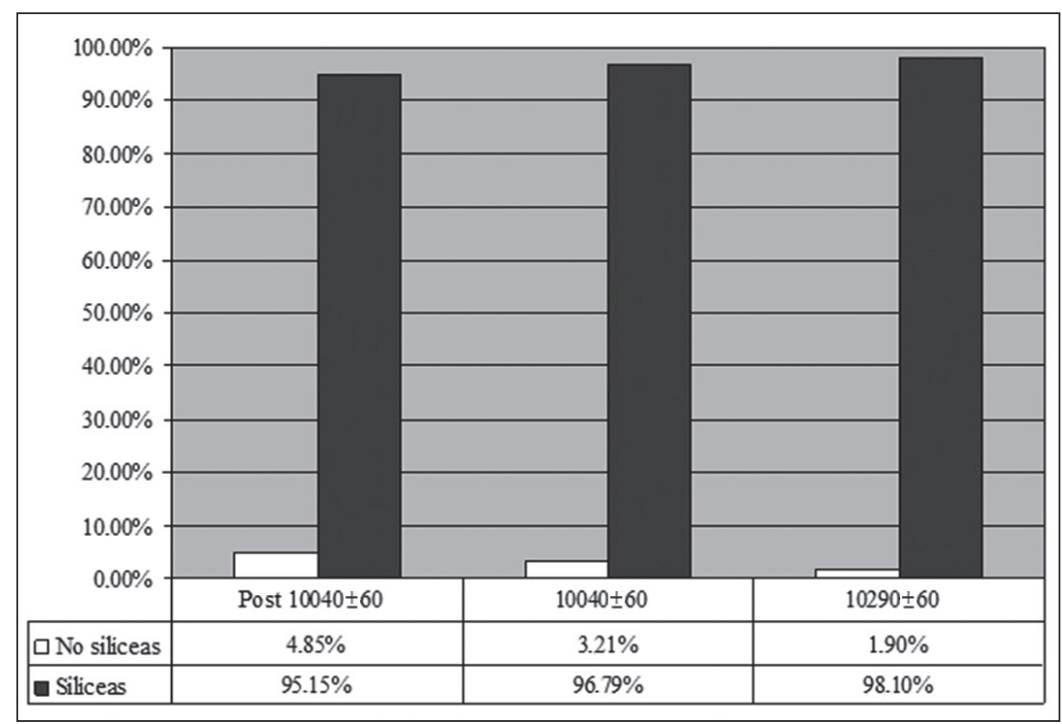

Figura 14. Frecuencia de materias primas por componente ocupacional en sitio 226-5.

badas, lo que indica que las actividades de talla fueron intensas en el interior del alero, aunque no podemos determinar con seguridad cuales momentos de ocupación fueron responsables de la depositación de esta considerable cantidad de restos.

En resumen, tenemos una dinámica espacial variable, donde existe un desplazamiento horizontal de los loci de talla lítica ocupando sectores exteriores y áreas cercanas al interior del alero. Al mismo tiempo, se aprecia una tendencia temporal desde una depositación poco intensa y de carácter disperso (10.290 $\pm 60 \mathrm{AP})$, a situaciones distribucionales donde se configuran loci de talla definidos, sea en la boca del alero (10.040 $\pm 60 \mathrm{AP})$ o en el borde exterior del mismo (post $10.040 \pm 60 \mathrm{AP}$ ). La movilidad horizontal de las áreas de actividad lítica sugiere que el sitio fue ocupado recurrentemente de manera transitoria.

Análisis tecnológico de materias primas y derivados líticos. Tal como se argumentó para el caso del sitio 228/230, la industria lítica del sitio 226-5 parece haber estado fundamentada en la utilización extensiva de materias primas silíceas y silicificadas de alta calidad, recursos líticos disponibles en áreas interiores cercanas a la costa de Taltal. Los siguientes análisis (tecnológico y de materias primas) fueron realizados solo sobre una muestra del total de derivados líticos recuperados en el sitio, equivalente a un porcentaje de $31.16 \%$ ( 1183 de 3796 piezas), ya que no se consideraron los materiales de aquellas unidades altamente disturbadas que conforman el componente ocupacional no determinado.

La representación frecuencial de las dos grandes agrupaciones de materias primas (silíceas y no silíceas), muestra una situación clara para todos los componentes ocupacionales, marcada por el predominio casi exclusivo de los materiales silíceos sobre otra clase de recursos líticos, en la conformación de los conjuntos líticos (Figura 14).

La abundante disponibilidad y fácil acceso a materias primas de alta calidad como las rocas silíceas, motivó que los grupos humanos fundamentaran la implementación de la tecnología lítica en dicha clase de recursos, utilizando solo de manera muy marginal y secundaria otros tipos de rocas como los basaltos, andesitas y granitos. Es sugerente el hecho que ya en fechas muy tempranas, anteriores a 10.000 años AP, se registrara un conocimiento cultural acabado del paisaje lítico alrededor de Taltal y una utilización intensiva de los mejores materiales líticos existentes en el área. Si la obtención de estos recursos líticos de alta calidad tiene que ver con estrategias de aprovisionamiento especializado o inserto (Binford 1979; Gould y Saggers 1985), es un problema que requiere un mayor grado de conocimiento de la estructura regional de recursos líticos, 


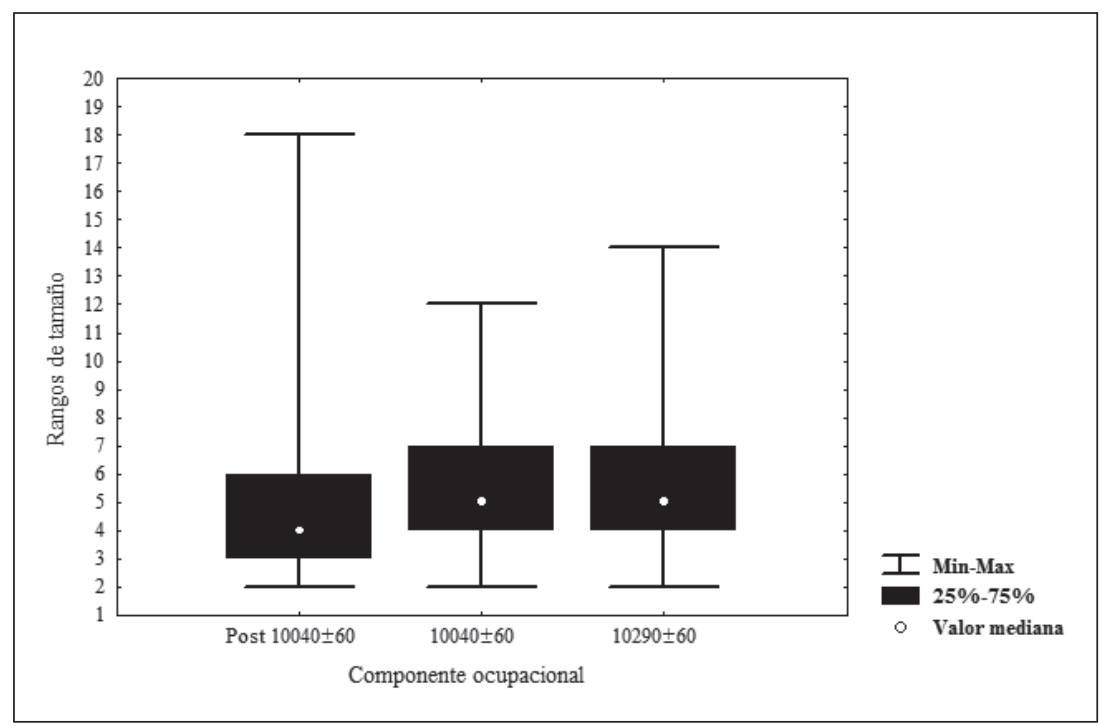

Figura 15. Distribución de rangos de tamaños en derivados por componente ocupacional en el sitio 226-5.

tarea aún pendiente para la zona que aquí nos ocupa. Pese a lo anterior, información proporcionada en algunos de los escasos estudios arqueológicos en el área de Taltal, permite constatar la existencia de extensos talleres líticos en las pampas, donde grupos costeros obtenían materias primas de alta calidad (Núñez 1984; Castelleti et al. 2010; Peralta et al. 2010). En dichos talleres se registran estructuras circulares señaladas como lugares de permanencia transitoria asociada al trabajo de talleres y canteras. Esta podría ser una evidencia que algunas conductas de aprovisionamiento lítico funcionaron dentro de una lógica de obtención dirigida, más que inserta en el desarrollo inmediato de otras actividades, por ejemplo de subsistencia.

Las distribuciones de tamaño para los distintos componentes ocupacionales (Figura 15), muestra que los momentos arcaicos tempranos $(10.290 \pm 60$ AP y $10.040 \pm 60 \mathrm{AP})$ se caracterizan por una alta concentración de casos en rangos métricos relativamente pequeños entre 04 y $07(20-35 \mathrm{~mm})$, con un valor para la mediana en el rango $05(25 \mathrm{~mm})$. A partir de estas distribuciones, postulamos que en estos conjuntos predomina ampliamente la generación de derivados líticos por actividades de talla avanzada de matrices (elaboración de instrumentos). Los pocos casos donde los derivados alcanzan mayor tamaño, hasta los rangos 12 y $13(60-65 \mathrm{~mm})$ no reflejarían un trabajo reductivo importante de núcleos en dichos momentos temporales, sino más bien la utilización de derivados más grandes para realizar distintas actividades, principalmente como instrumentos de filos vivos o eventuales matrices para confeccionar diversas herramientas líticas.

La situación es parecida en el componente post $10.040 \pm 60$ $\mathrm{AP}$, con un predominio mayoritario de actividades de talla avanzadas sobre iniciales, aunque la mayor concentración de casos ocurre en rangos métricos menores entre 03 y 06 $(15-30 \mathrm{~mm})$ con valor para la mediana en el rango 04 (20 $\mathrm{mm})$. En conclusión, estamos frente a conjuntos donde predominaron ampliamente las actividades de talla concernidas con el desbaste de matrices y la manufactura de instrumentos, indicando que este componente ocupacional se organizó de manera semejante a un campamento residencial de carácter transitorio.

En un cuadro marcado por actividades de talla lítica relacionadas fuertemente con la manufactura de instrumentos, conviene preguntarse cuáles eran las preferencias de elaboración, evaluando las variaciones en la plataforma de percusión como indicador de la orientación del proceso reductivo en este contexto lítico (Figura 16). Como vemos, los tres componentes ocupacionales evaluados se comportan prácticamente de igual manera, con un claro predominio de platafor- 


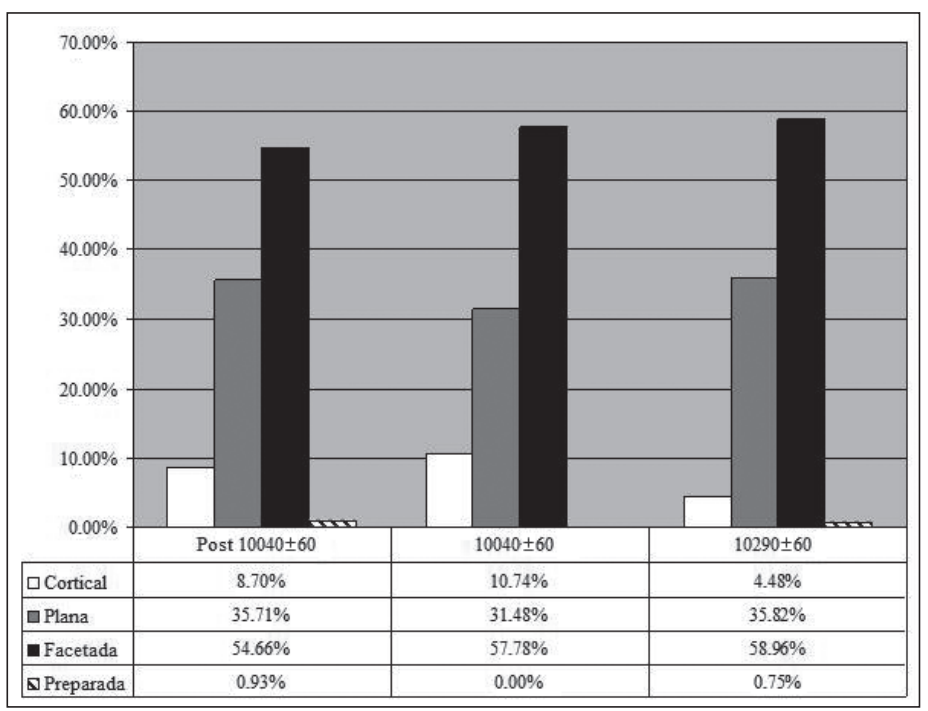

Figura 16. Frecuencia de morfologías de plataformas por componente ocupacional en el sitio 226-5.

\begin{tabular}{|c|c|c|c|c|c|}
\hline Categoría morfofuncional & No determinado & Post $10.040 \pm 60$ & $10.040 \pm 60$ & $10.290 \pm 60$ & Total \\
\hline Puntas proyectil & $17(27.42 \%)$ & $5(20.0 \%)$ & $6(12.50 \%)$ & $3(16.67 \%)$ & $31(20.53 \%)$ \\
\hline Bifaces & $15(24.19 \%)$ & $6(24.0 \%)$ & $10(20.83 \%)$ & $4(22.22 \%)$ & $35(23.18 \%)$ \\
\hline Instrumentos lasca bifacial & $22(35.48 \%)$ & $8(32.0 \%)$ & $13(27.08 \%)$ & $8(44.44 \%)$ & $51(33.77 \%)$ \\
\hline Cuchillos & $5(8.06 \%)$ & $5(20.0 \%)$ & $10(20.83 \%)$ & $1(5.56 \%)$ & $21(13.91 \%)$ \\
\hline Denticulados o muescas & $3(4.84 \%)$ & $0(0.0 \%)$ & $0(0.0 \%)$ & $2(11.11 \%)$ & $5(3.31 \%)$ \\
\hline Leznas & $0(0.0 \%)$ & $0(0.0 \%)$ & $2(4.17 \%)$ & $0(0.0 \%)$ & $2(1.32 \%)$ \\
\hline Raspadores dorso alto & $0(0.0 \%)$ & $0(0.0 \%)$ & $4(8.34 \%)$ & $0(0.0 \%)$ & $4(2.65 \%)$ \\
\hline Tajadores & $0(0.0 \%)$ & $1(4.0 \%)$ & $0(0.0 \%)$ & $0(0.0 \%)$ & $1(0.66 \%)$ \\
\hline Núcleos & $0(0.0 \%)$ & $0(0.0 \%)$ & $1(2.08 \%)$ & $0(0.0 \%)$ & $1(0.66 \%)$ \\
\hline Manos y molinos planos & $0(0.0 \%)$ & $0(0.0 \%)$ & $2(4.17 \%)$ & $0(0.0 \%)$ & $2(1.32 \%)$ \\
\hline Total & $62(100 \%)$ & $25(100 \%)$ & $48(100 \%)$ & $18(100 \%)$ & $153(100 \%)$ \\
\hline
\end{tabular}

Figura 17. Frecuencia de categorías morfofuncionales por componente ocupacional en el sitio 226-5.

mas de morfologías facetadas y planas, lo que reafirma la idea de que las actividades de talla lítica realizadas correspondieron a la manufactura de instrumentos trabajados bifacialmente. En este sentido, la presencia minoritaria de plataformas corticales sugiere, más que la presencia de desbaste de núcleos, el trabajo de matrices con remanentes corticales.

La importancia de la reducción bifacial en este tipo de contextos nos habla del transporte y utilización frecuente de esta clase de instrumentos. Recordemos que en varios contextos de caza y recolección, uno de los objetos líticos infaltables en el equipo artefactual son los bifaces, los cuales pueden operar tanto como instrumentos, preformas o núcleos, dependiendo del contexto situacional en el cual se desenvolvía el grupo (Kelly 1988).

Análisis morfofuncional de los instrumentos líticos. La representación de categorías morfofuncionales en los distintos componentes ocupacionales muestra una situación compartida en la cual predominan los instrumentos bifaciales por sobre otras categorías artefactuales (Figura 17). 
Este cuadro es coherente con lo observado en el análisis tecnológico de derivados, donde se destaca la importancia de la reducción bifacial en los conjuntos estudiados. Tres son las categorías morfofuncionales manufacturadas bifacialmente, que destacan dentro del conjunto y cuya situación discutiremos aquí.

Los instrumentos bifaciales más frecuentes corresponden a las categorías puntas de proyectil, bifaces y un conjunto de instrumentos elaborados a partir de lascas de desbaste bifacial, incluyendo raspadores -raederas, cuchillos y raspadores de borde circular-. La importancia que tiene la manufactura de estos instrumentos, pone en el tapete el papel que juegan estas piezas para los grupos cazadores recolectores. Recurriendo al modelo de Kelly (1988), acerca de las tres dimensiones tecnológicas y funcionales que presentan los bifaces, el procesamiento bifacial de las materias primas permite reforzar la disponibilidad de material lítico, ayudando a prevenir la escasez de suministros tecnológicos durante la realización de las actividades de subsistencia.

Para los distintos componentes determinados, tenemos una situación común marcada por la utilización de instrumentos manufacturados mediante esta estrategia reductiva, donde las categorías más frecuentes son las de instrumentos sobre lascas bifaciales, los bifaces y las puntas de proyectiles y cuchillos.

Este cuadro nos dice que en los componentes arcaicos existe un predominio relativo de aquellas actividades orientadas al procesamiento de recursos, donde los instrumentos elaborados a partir de lascas de desbaste bifacial fueron dirigidos a la realización de diversas actividades domésticas. Las piezas incluidas en la categoría bifaces corresponden a fragmentos de núcleos bifaciales y preformas, representando el trabajo de este tipo de matrices en la locación. Por su parte, la situación de las puntas de proyectil resulta especial, puesto que la mayoría corresponden a piezas que presentan grados de formatización y terminación insuficientemente desarrollados, propios de talladores aprendices con distintos grados de pericia técnica (Dawe 1997; Politis 1999; Galarce 2008; Jackson 2008). Las puntas de proyectil que fueron manufacturadas por talladores experimentados suelen presentarse fragmentadas, indicando su descarte post utilización en el sitio.
De la situación representada por los instrumentos líticos, inferimos básicamente un desarrollo de actividades de talla lítica en un contexto de carácter residencial y doméstico, marcadas por el predominio de una amplia diversidad de instrumentos cuya morfología y configuración de los bordes activos sugiere un fuerte desarrollo de las actividades de procesamiento de recursos, sobre todo en el caso de instrumentos manufacturados a partir de lascas de adelgazamiento bifacial.

Pese a compartir características comunes, los tres componentes ocupacionales presentan diferencias funcionales entre sí. El componente de $10.040 \pm 60 \mathrm{AP}$, es la ocupación con mayor diversidad funcional y con más categorías morfofuncionales representadas, mientras que lo contrario ocurre con la ocupación más temprana (10.290 \pm 60 AP) y con la ocupación post $10.040 \pm 60 \mathrm{AP}$. Dado que, de acuerdo con la distribución de restos líticos, en el componente intermedio la actividad de talla principal se da en la boca del alero rocoso, podemos inferir que el sitio funcionó como un lugar claramente orientado a la realización de actividades residenciales centradas en el alero y sus alrededores inmediatos (Casteletti et al. 2010). La menor diversidad funcional para los otros componentes ocupacionales sugiere que, si bien existe un predominio de actividades residenciales a lo largo de la ocupación del asentamiento, éstas configuran un campamento centrado en el alero propiamente tal solo en el componente de $10.040 \pm 60$ AP. Para el componente más temprano, la baja cantidad de restos, tanto instrumentos como derivados líticos, la no estructuración clara de áreas de actividad lítica y una menor diversidad funcional sugiere la presencia de un campamento de uso residencial o logístico, de carácter altamente transitorio. El componente post $10.040 \pm 60$ $\mathrm{AP}$, aunque se comporta de manera similar al anterior, muestra la configuración del principal locus de talla lítica en los bordes externos del área de excavación, indicando que las áreas de actividad en el sitio y, por tanto, el lugar de desarrollo de las actividades residenciales se encuentra alejado del bloque rocoso donde se emplaza el alero.

\section{$\leadsto$ Conclusiones}

Varios aspectos destacables permiten hacer una interpretación sintética del estudio de los contextos líticos de los sitios $228 / 230$ y $226-5$. 
Un primer aspecto se refiere a la estructura distribucional que presenta la actividad talla y descarte lítico en los sitios estudiados. Estos se comportan como lugares donde las actividades de talla y descarte configuraron loci bien definidos y discretos, en los que se llevó a cabo intensa actividad reductiva en materias primas de alta calidad. Para el sitio 228/230, estos contextos se encuentran asociados a grandes afloramientos rocosos presentes en el lugar. Esta concentración de la actividad lítica permanece como una constante a lo largo de la ocupación del sitio, aunque las intensidades de trabajo reductivo varían de más a menos, transcurriendo desde el componente inicial de ocupación a los más tardíos identificados.

Para el sitio 226-5, la información distribucional de restos depositados muestra una situación más variable, donde el lugar de emplazamiento de los loci de talla se desplaza horizontalmente entre la boca del alero rocoso y el perímetro externo del área de excavación, entre los distintos momentos de ocupación del lugar. Las intensidades de talla lítica cambian también cronológicamente, con el primer componente (10.290 $\pm 60 \mathrm{AP})$ que muestra una situación marcada por escasa concentración de materiales y la no configuración de loci claramente definidos. En una segunda situación (10.040 $\pm 60 \mathrm{AP})$, existe la conformación de un locus denso de talla lítica en la boca del alero y la presencia de otras áreas menos definidas de concentración en el exterior del mismo (talud). Por último, para el componente post $10.040 \pm 60 \mathrm{AP}$, la mayor concentración de trabajo lítico se registra en el exterior del alero (talud), apreciándose un relativo abandono del sector de la boca, como área principal de actividad.

Los patrones distribucionales de restos líticos en ambos sitios permiten sostener que en $228 / 230$ se observa una utilización recurrente de los espacios físicos para desarrollar actividades de talla lítica, mientras que en el caso de 226-5 se aprecia una rotación espacial de las áreas de trabajo reductivo entre los distintos momentos de ocupación del lugar.

Otro aspecto que debemos considerar se refiere a las características tecnológicas de los contextos líticos de estos dos sitios. Se observa una situación compartida entre ambos, marcada por la utilización extensiva de materias primas silíceas de alta calidad, las que parecen haber sido obtenidas desde fuentes de aprovisionamiento situadas en el área interior aledaña a la costa (pampas). La escasa utilización de materiales no silíceos refuerza la idea que los grupos respaldaron el funcionamiento de sus sistemas tecnológicos en los abundantes y accesibles recursos de alta calidad que se presentan en áreas cercanas. Este punto es bastante coincidente con investigaciones más amplias desarrolladas en contextos arqueológicos del área costera de Taltal (Castelleti y Maltrain 2010; Castelleti et al. 2010).

Asociado a la anterior situación, vemos que la tecnología lítica predominante en los conjuntos de ambos sitios es la reducción bifacial de matrices. Esto indica que los grupos transportaron a los asentamientos costeros matrices bifaciales previamente trabajadas, probablemente en los mismos lugares de obtención, a partir de las cuales eran elaborados instrumentos directamente o a partir de lascas extraídas de dichas matrices. La información tecnológica aportada sugiere fuertemente la operación de estrategias de aprovisionamiento lítico dirigido (sensu Gould y Saggers 1985), más que insertos (Binford 1979; Binford y Stone 1985), aunque enfatizando el procesamiento bifacial de las materias primas de alta calidad. El empleo extensivo de matrices bifaciales en estos contextos permite aprovechar de modo más eficiente la disponibilidad de materias primas lejos de las fuentes de aprovisionamiento (Kelly 1988), por lo que tal conducta será favorecida en situaciones con presencia de grupos móviles y donde los recursos de subsistencia son especialmente incongruentes con los recursos líticos necesarios para lograr su obtención, como ocurre con los grupos cazadores recolectores de esta parte de la costa norte de Chile.

Respecto a la funcionalidad de los instrumentos descartados, se observa una fuerte correspondencia entre formas de procesamiento de las materias primas, básicamente a través de reducción bifacial, y los tipos de instrumentos que fueron elaborados por estos grupos, donde la representación de piezas bifaciales o elaboradas a partir de lascas de desbaste bifacial es mayoritaria dentro de los conjuntos de instrumentos. Aunque esta es una situación compartida entre ambos sitios, existen diferencias claras en los énfasis funcionales observados en cada uno de los contextos. De esta manera, mientras los conjuntos artefactuales del sitio $228 / 230$ muestran un predominio frecuencial de las puntas de proyectil sobre otras categorías morfofuncionales, en los conjuntos del sitio 226-5 el tipo más frecuente de instrumento relacionado con la 


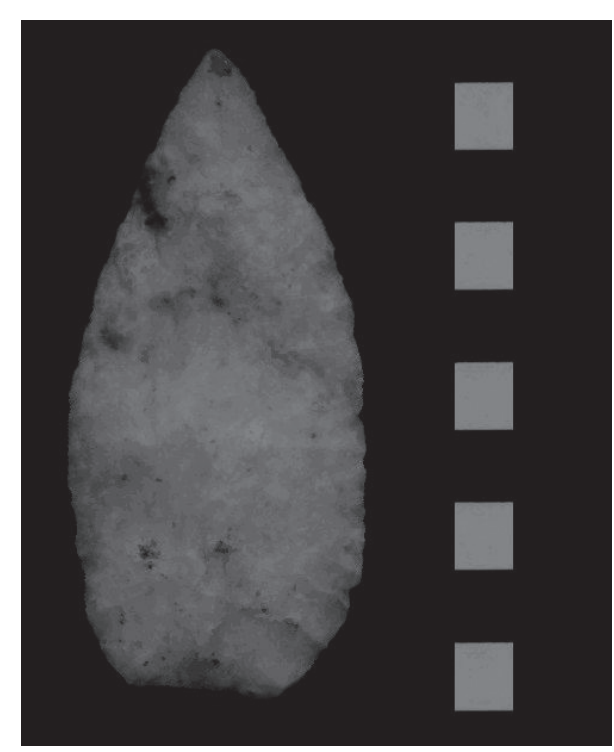

Figura 18. Hoja bifacial lanceolada en calcedonia blanca, sitio 228/230 asociada a inhumación.

bifacialidad son aquellos elaborados a partir de lascas extraídas desde grandes matrices bifaciales, donde su funcionalidad se refiere más al procesamiento de recursos obtenidos mediante la caza, que al desarrollo de tareas involucradas en la obtención directa de este tipo de recursos. Por lo tanto, de manera similar que estudios desarrollados en el área (Castelleti et al. 2010), tendemos a inferir un comportamiento funcional propio de un campamento de caza para el sitio $228 / 230$ y una funcionalidad propia de campamento residencial transitorio para el sitio 226-5.

Por último, conviene hacer mención a dos aspectos fuertemente vinculados a la organización social de los grupos que presenta la tecnología bifacial en ambos asentamientos. Nos referimos a la asociación de piezas bifaciales y un entierro humano en el sitio $228 / 230$ y a la representación de conductas de aprendizaje de talla lítica en el sitio 226-5.

En el caso del sitio 228/230, las excavaciones permitieron registrar un entierro humano secundario asociado a un bloque rocoso, en el cual se encontró como parte de las ofrendas del difunto, una hoja bifacial grande (Figura 18) y una punta pedunculada, ambas piezas completas $y$ elaboradas en la misma materia prima, una calcedonia de color blanco con algunas motas grisáceas (Castelleti et al. 2004) La presencia de estas piezas, aparentemente no

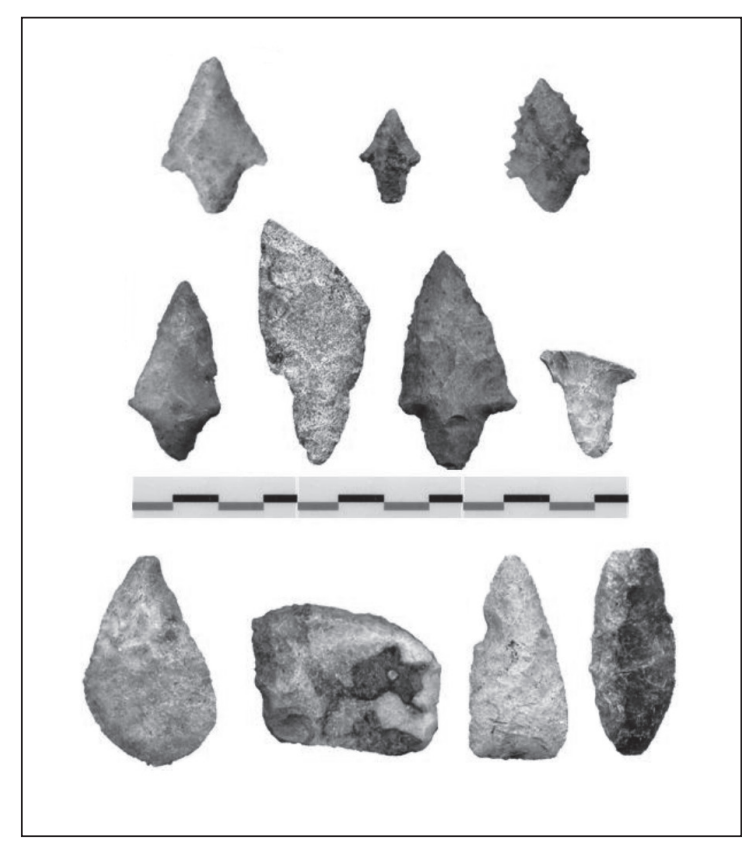

Figura 19. Puntas de proyectil e instrumentos sobre lascas bifaciales, sitio 226-5. Algunas piezas más pequeñas y asimétricas corresponden a ejemplos de instrumentos manufacturados por talladores aprendices.

utilizadas, en un contexto funerario demuestra, a nuestro juicio, la importancia que tenía para los grupos del Arcaico Medio y Tardío de la zona el hecho de elaborar piezas líticas bifaciales, las cuales podían funcionar también dentro de una esfera de interacción social y simbólica representada en el ritual funerario.

Otro aspecto se registra en el sitio 226-5, donde varios ejemplares de puntas pedunculadas arcaicas tempranas parecen corresponder a piezas elaboradas por aprendices de talla junto a piezas claramente trabajadas por talladores con alto grado de pericia (Figura 19). Al respecto, resulta esperable que, en contextos residenciales como este sitio, se registren conductas de aprendizaje dado que los individuos de un grupo cazador recolector deben estar familiarizados desde pequeños con las formas sociales de manufacturar instrumentos, que después van a ser claves para su desempeño como cazadores adultos. No es de extrañar entonces, encontrar en ciertos contextos arqueológicos la presencia de individuos aprendices, sobre todo niños, quienes a través de sus juegos se entrenan para ser cazadores experimentados (Dawe 1997; Politis 1999). 
En este sentido, el registro de actividad de aprendices en el contexto 226-5 se entiende en razón del carácter más residencial de este sitio dentro de su sistema de asentamiento. Por su parte, la ausencia de indicadores líticos de aprendices en el contexto 228/230 puede deberse a su carácter funcionalmente más específico de esta locación, vinculada con la caza y recolección en borde litoral, dentro de las estrategias de asentamiento de los grupos de finales del Arcaico Medio en el área.
Finalmente, no cabe más que recalcar la importancia de estudiar mayor número de contextos de cazadores recolectores en la costa del Norte Grande, donde pese al gran potencial arqueológico que se visualiza, por ejemplo en estos sitios, es escaso e irregular el trabajo investigativo realizado hasta la fecha. Esperamos que este estudio sirva para ayudar al desarrollo de aproximaciones más sistemáticas a la problemática arqueológica de esta área de la costa pacífica sudamericana.

\section{* Referencias citadas}

ANDREFSKY, W., 1998. Lithics: Macroscopic approaches to analysis. Manuals Cambridge in Archaeology. Cambridge University Press, Cambridge.

2001. Emerging directions in debitage analysis. En Lithic debitage: Context, form, meaning, W. Andrefsky (Ed.), pp. 2-12. The University of Utah Press, Salt Lake City.

2008. An introduction to stone tool life history and technological organization. En Lithic technology: Measures of production, use and curation, W. Andrefsky (Ed.), pp. 3-22. Cambridge University Press, Cambridge.

ARAGÓN, E. y N. FRANCO. 1997. Características de rocas para la talla por percusión y propiedades petrográficas. Anales del Instituto de la Patagonia (Serie Ciencias Humanas) 25: 87-199.

BATE, L., 1971. Material lítico: Metodología de clasificación. Noticiario Mensual del Museo Nacional de Historia Natural Año XVI (181182): 3-24.

BERDICHEWSKY, B., 1962. El Precerámico de Taltal y sus correlaciones. Publicación del Centro de Estudios Antropológicos 16.

BINFORD, L., 1979. Organization and formation processes: Looking at curated technologies. Journal of Anthropological Research 35(3): $255-273$.

BINFORD, L. y N. STONE. 1985. "Righteous rocks" and Richard Gould: Some observations on misguided "debate". American Antiquity 50(1): 151-153.

BIRD, J., 1988 [1943]. Excavaciones en el norte de Chile. Ediciones Universidad de Tarapacá, Arica.

BRADBURY, A. y P. CARR, 1999. Examining stage and continuum models of flake debris analysis: An experimental approach. Journal of Archaeological Science 26: 105-116.
CAPDEVILLE, A., 1921. Notas acerca de la arqueología de Taltal. Boletín de la Academia Nacional de Historia, vol. II (5), Quito.

CASTELLETI, J. y G. MALTRAIN, 2010. El Formativo de Taltal y el patrón de asentamiento local. Actas del XVII Congreso Nacional de Arqueología Chilena (2006), vol. 1, pp. 165-176. Ediciones Kultrún, Valdivia.

CASTELLETI, J., O. REYES, H. VELÁSQUEZ, I. MARTÍNEZ, V. TREJO, D. BAUDET, P. GALARCE, G. MALTRAIN, C. BELMAR, L. QUIROZ, M. DE LA MAZA y J. OGALDE, 2004. Proyecto mejoramiento y construcción Ruta 1, tramo Taltal-Paposo, II Región, Chile, Rescate sitios arqueológicos 226-5 y 228/230. Cuerpo Militar del Trabajo.

CASTElleti, J., O. REYES, G. MALTRAin, I. MARTÍNEZ, P. GALARCE, H. VELÁSQUEZ y J. OGALDE, 2010. Ocupaciones en abrigos rocosos en la costa de Taltal: Patrón de uso del espacio desde momentos holocénicos tempranos. Actas del XVII Congreso Nacional de Arqueología Chilena (2006), vol. 2, pp. 685-695. Ediciones Kultrún, Valdivia.

CORNEJO, L. y P. GALARCE, 2010. C index: Dimensioning the expedient/curative continuum in lithic technology. Chungar. Revista Chilena de Antropología 42(2): 393-404.

DAWE, B., 1997. Tiny arrowheads: Toys in the toolkit. Plains Anthropologist 42(161): 303-318.

DE SOUZA, P., 2003. Tecnología lítica y sistemas de asentamiento de los cazadores recolectores del Arcaico Temprano y Medio en la cuenca superior del río Loa. Memoria para optar al título de Arqueólogo. Departamento de Antropología, Facultad de Ciencias Sociales, Universidad de Chile. Santiago.

FUENTES, F., M. BISKUPOVIC, J. CASTELLETI y M. RETAMALES (Eds.), 2010. Tradiciones de tierra y mar: Antiguos pescadores, mariscadores y cazadores del Semiárido. Andros Impresores, Santiago. 
GALARCE, P., 2004. Cazadores recolectores tempranos en la costa sur del Semiárido: Aprovisionamiento y procesamiento de recursos líticos. Memoria para optar al título de Arqueólogo. Departamento de Antropología, Facultad de Ciencias Sociales, Universidad de Chile, Santiago.

2005. Aproximación experimental a la elaboración de instrumentos: Un aporte al estudio tecnofuncional de los conjuntos líticos arqueológicos. Actas del XVI Congreso Nacional de Arqueología Chilena, pp. 55-64. Tomé.

2008. Aprendizaje y talla lítica en sociedades prehistóricas: Contextos sociales y correlatos materiales. Puentes hacia el pasado: Reflexiones teóricas en arqueología, D. Jackson, D. Salazar y A. Troncoso (Eds.), pp. 93-110. Universidad de Chile, Santiago.

GOULD, R. y S. SAGGERS, 1985. Lithic procurement in Central Australia: A closer look at Binford's idea of embeddedness in archaeology.American Antiquity 50(1): 117-136.

JACKSON, D., 1991. Raspadores de vidrio en Dinamarquero: Reflejo de una encrucijada cultural. Anales del Instituto de la Patagonia 20: 169-203.

1993. Datación radiocarbónica para una adaptación costera del Arcaico Temprano en el Norte Chico, comuna de Los Vilos. Boletín de la Sociedad Chilena de Arqueología 16: 28-31.

2008. Infancia y arqueología: Hacia la construcción de un marco conceptual y expectativas arqueológicas. Puentes hacia el pasado: Reflexiones teóricas en arqueología, D. Jackson, D. Salazar y A. Troncoso (Eds.), pp. 111-122. Universidad de Chile, Santiago.

JACKSON, D. y C. MÉNDEZ, 2005. Primeras ocupaciones humanas en la costa del Semiárido de Chile: Patrones de asentamiento y subsistencia. Actas del XVI Congreso Nacional de Arqueología Chilena, pp. 493-502. Museo de Historia Natural de Concepción, Concepción.

KELLY, R., 1988. The three sides of a biface. American Antiquity 53(4): 717-734.

LLAGOSTERA, A., 1979. Ocupación humana en la costa norte de Chile asociada a peces locales extintos y a litos geométricos: $9680 \pm 160$ AP. Actas del VII Congreso Nacional de Arqueología Chilena (1977), pp. 93-114. Ediciones Kultrún, Valdivia.

LLAGOSTERA, A., R. WEISNER, G. CASTILLO, M. CERVELLINO y M. A. COSTA, 2000. El Complejo Huentelauquén bajo una perspectiva macroespacial y multidisciplinaria. Contribución Arqueológica 5: 461-480.

LUEDTKE, B., 1979. The identification of sources of chert artifacts. American Antiquity 44(4): 744-756.
1992. An archaeologist's guide to chert and flint. Archaeological Research Tools 7. Institute of Archaeology, University of California, Los Angeles.

MANSUR, M., 1997. Functional analysis of polished stone-tools: Some considerations about the nature of polishing. En Siliceous rocks and culture, A. Ramos-Millán y M. Bustillo (Eds.), pp. 465486. Universidad de Granada, Granada.

NÚÑEZ, L., 1984. Secuencia de asentamientos prehistóricos del área de Taltal. Futura 8: 28-76.

OYARZÚN, A., 1917. Estación paleolítica de Taltal. Publicación del Museo de Etnología de Chile, vol. 1. Santiago.

PERALTA, P., C. GONZÁLEZ, C. WESTFALL y G. SANTANDER, 2010. Primeras aproximaciones sobre la arqueología de Pampa Austral: Explotación y tecnología lítica al interior de la Región de Atacama (Chile). Actas del XVII Congreso Nacional de Arqueología Chilena (2006), vol. 1, pp. 297-306. Ediciones Kultrún, Valdivia.

PIEL-DESRUISSEAUX, J., 1989. Instrumental prehistórico: Forma, fabricación y utilización. Masson, Barcelona.

POLITIS, G., 1999. La actividad infantil en la producción del registro arqueológico de cazadores recolectores. Revista do Museo de Arqueología y Etnologia 3: 263-283.

SCHIAPPACASSE, V. y H. NIEMEYER, 1968. Noticia y comentario de dos fechas radiocarbónicas para un sitio arqueológico en Guanaqueros, prov. de Coquimbo. Noticiero Mensual del Museo Nacional de Historia Natural 147:3-6.

1986. El Arcaico en el norte semiárido de Chile. Chungara 1617: 95-98.

SEMENOV, S., 1981. Tecnología prehistórica. Akal Editores, Barcelona.

SHOTT, M., 1993. The Leavitt site: a Parkhill phase Paleoindian occupation in Central Michigan. Memoirs Museum of Anthropology, University of Michigan 25. Ann Arbor.

SULLIVAN, A. y K. ROZEN, 1985. Debitage analysis and archaeological interpretation. American Antiquity 50(4): 755-779.

UHLE, M., 1916. Sobre la estación paleolítica de Taltal, una carta y un informe. Publicación del Museo de Etnología y Antropología de Chile, vol. 1. Imprenta Universo, Santiago.

WEISNER, R., A. LLAGOSTERA, G. CASTILLO y M. CERVELLINO, 2000. El sitio arqueológico Huentelauquén en el contexto del Arcaico Temprano de la porción sur del Semiárido de Chile. Contribución Arqueológica 5: 579-620.

WHITTAKER, J., 1994. Flintknapping: Making and understanding stone tools. University of Texas Press, Austin. 